\title{
Structural Development of Nanoparticle Dispersion during Drying in Polymer Nanocomposite Films
}

Sunhyung Kim ${ }^{1}$, Kyu Hyun ${ }^{2}$, Bernd Struth $^{3}$, Kyung Hyun $\mathrm{Ahn}^{4, *}$ and Christian Clasen ${ }^{1, *}$

${ }^{1}$ Department of Chemical Engineering, KU Leuven, Celestijnenlaan 200F, B-3001, Heverlee, Belgium

${ }^{2}$ School of Chemical and Biomolecular Engineering, Pusan National University, JangjeonDong 30, Busan 609-735, Republic of Korea

${ }^{3}$ DESY, Notkestrasse 85, D-22607 Hamburg, Germany

${ }^{4}$ School of Chemical and Biological Engineering, Institute of Chemical Process, Seoul National University, Seoul 151-744, Republic of Korea

*Corresponding Authors:

christian.clasen@,cit.kuleuven.be +32-16-322354

ahnnet@snu.ac.kr, +82-2-880-8322 


\begin{abstract}
Dispersions of nanoparticles (NPs) in a polymer matrix are a key element to set various properties of solution-cast polymer nanocomposite films. While the dispersion state of NPs in nanocomposite films has been extensively studied over the last decades, the structural development during drying and the relation of liquid and solid structure still remains poorly understood. In the present work, we study how NP dispersions develop during drying in polymer nanocomposite films, particularly focusing on the role of particle-polymer interaction in the structural development. Aqueous dispersions of nano-silica and poly(vinyl alcohol) are used as a model NP/polymer mixtures, where the particle-polymer interaction can systemically be varied via the $\mathrm{pH}$. A novel vertical small angle X-ray scattering technique enables us to directly observe the development of NPs dispersion during drying. At a high $\mathrm{pH} 10$, where silica particles have a poor affinity to PVA, SAXS intensity evolution shows phase separation during drying, resulting in the formation of dense aggregates of bare particles in the solid film. On the other hand, at a low $\mathrm{pH} 3$, where silica particles have a good affinity with PVA, the SAXS data indicates a gradual densification of the NPs while maintaining a constant inter-particle distance, which is originating from adsorbed PVA. The resulting solid film after drying exhibits an improved dispersion of NPs. The evaluation of the inter-particle interaction suggests that the adsorbed polymer plays generally a key role in the uniform distribution of NPs in solid films, as it sterically stabilizes NPs over short ranges during all drying stages whereas depletion attraction dominates at longer ranges.
\end{abstract}

Keywords: Polymer nanocomposite film, drying, nanoparticle dispersion, particle-polymer interaction 


\section{Introduction}

Polymer nanocomposite films prepared from solvent evaporation have received considerable interest over the last decades owing to the versatility of formulation and wide applicability of the processes. Recent applications include catalytic electrodes in fuel cells ${ }^{1}$, organic photovoltaic solar cells ${ }^{2}$, solid electrolytes for fuel cells ${ }^{3}$ or $\mathrm{Li}$-ion batteries ${ }^{4}$, ceramic capacitors $^{5}$, membranes for gas separation ${ }^{6}$, and antireflective coatings ${ }^{7}$. To realize the required properties in those applications, it is important to control the dispersion of nanoparticles (NPs) in the polymer matrix.

Controlling NP dispersion in polymer matrices is one of the most actively studied subjects in the field of polymer/colloid science and relevant industries ${ }^{8-11}$. A well-known methodology to obtain a desired state of NP dispersion in polymer nanocomposites is to control the interparticle interaction. One possible route for this is to select a suitable solvent character or quality to tune the physicochemical affinity between the particle surface and the hosting polymer. Several studies report on the effect of the solvent on this affinity, and the resulting NP dispersion in the solid nanocomposite ${ }^{12-14}$. When casting onto a substrate, solvent evaporates and the liquid film inevitably experiences a significant volume change and structural evolution of the dispersion of the NPs. More specifically from the viewpoint of particle interaction, when drying occurs, solvent evaporation increases the volume fraction of particles, polymers and additives, which results in a significant change in the particle interactions and the resulting NP dispersion structure. In the case of a poor NP-polymer affinity, depletion attraction by non-adsorbed polymer or van der Walls attraction can result in NP aggregation in the solid film after drying ${ }^{12,14,15}$. Conversely, in case of a good affinity between NPs and the hosting polymer matrix, the polymers may adsorb on the NP surface and introduce a steric repulsion, thus prevent NP aggregates in the final solid film ${ }^{12,16,17}$. Therefore, a deeper understanding of the evolution of interparticle interaction and the impact 
on the structural development of the NP dispersion during drying is required in order to control and achieve a desired NP dispersion in the dried polymer nanocomposite films.

While there is a considerable amount of studies on the dispersion state of NPs in the final polymer nanocomposite, the understanding on how NP dispersions are formed and evolve during drying is still lacking. Recent studies have observed the structural evolution of NP dispersion during solvent evaporation by means of in-situ or ex-situ small angle x-ray scattering techniques ${ }^{18-25}$, but only dealt with colloid systems in the absence of polymers, which prevented to consider the affinity between particle and hosting polymer. Very recently, attempts have been made to understand the formation of NP dispersions in polymer films during drying ${ }^{15,26}$, but the role of the affinity between particle and hosting polymer, and the evolution of inter-particle interaction during drying was not adequately considered.

In the present study, we investigate the evolution of NP dispersion in polymer nanocomposite films during drying, with a particular focus on the role of the affinity of particle and the hosting polymer on the development of inter-particle interaction and resulting formation of NP dispersion. We directly observe the development of NP dispersion during drying by means of synchrotron vertical small angle X-ray scattering (vertical SAXS), where a vertical beam is directed through the film drying on a horizontal plate (see Fig. 1) ${ }^{27}$. Vertical SAXS enables the direct observation of the structural development of NP dispersion during drying without any gravity-related restrictions, which conventional (horizontal) synchrotron beams cannot achieve ${ }^{18-25}$. We use aqueous dispersion of nano-silica and poly(vinyl alcohol) as a model NP/polymer solution, where the particle-polymer interaction is systematically varied via the $\mathrm{pH}$. The evaluation of the inter-particle interaction at different drying stages allows us to explain the respective development of NP dispersion at two selected $\mathrm{pH}$ values of 3 and 10 via steric repulsion and depletion attractions, and their dominance at different length scales. 


\section{Sample preparation and experimental method}

Sample preparation Silica and poly(vinyl alcohol) (PVA) mixtures were prepared by mixing colloidal silica, PVA in solution, and deionized water. $30 \mathrm{wt} \%$ charge-stabilized colloidal silica (Ludox ${ }^{\circledR}$ HS-30, Aldrich, density $\rho=2.2 \times 10^{3} \mathrm{~kg} / \mathrm{m}^{3}$ according to the supplier) was used without further treatment. The average diameter $d$ of the silica particles was determined from the SAXS intensity to be $15 \mathrm{~nm}$, with $\sigma / d=0.145$, where $\sigma$ is the width parameter of the Schulz distribution function ${ }^{27}$. A 15 wt $\%$ aqueous solution of PVA (Aldrich, $M_{\mathrm{n}}=31-51 \times$ $10^{3} \mathrm{~g} / \mathrm{mol}$, degree of hydrolysis $87-88 \%$, and density $\rho=1.2 \times 10^{3} \mathrm{~kg} / \mathrm{m}^{3}$ according to the supplier) was prepared by dissolving PVA at $80^{\circ} \mathrm{C}$ for $2 \mathrm{~h}$. After preparing a silica/PVA mixture with $10 \mathrm{wt} \%$ silica and $4 \mathrm{wt} \%$ PVA, the $\mathrm{pH}$ was adjusted by adding $0.3 \mathrm{M} \mathrm{HCl}$ solution to the suspension (which was initially at $\mathrm{pH} 10$ ). When decreasing the $\mathrm{pH}$ to 3 , the suspension abruptly becomes turbid. The radius of gyration $R_{\mathrm{g}}$ of PVA was set to be $6 \mathrm{~nm}$ based on the literature ${ }^{28}$ and assumed to be independent of the $\mathrm{pH}$. All measurements of liquid structure as well as the drying experiments were performed after $12 \mathrm{~h}$ of magnetic stirring of the final composition at room temperature.

Liquid structure The amount of adsorbed PVA on the silica surface $\Gamma$, zeta potential $\zeta$, and zero shear viscosity $\eta_{0}$ were determined for all investigated suspensions. The amount of adsorbed PVA on the silica surface $\Gamma$ was determined via centrifugation. Particles bearing adsorbed PVA were separated from the suspension by centrifuging at $23140 \mathrm{~g}$ for $1 \mathrm{~h}$ and the concentration of PVA in the supernatant was determined by weighing the polymer after evaporation of the solvent water at $70^{\circ} \mathrm{C}$ for 24 hours. We experienced difficulties with sedimentation at polymer concentrations of $\phi_{\mathrm{PVA}}=4 \mathrm{wt} \%$ (the actual polymer concentration 
used for the drying experiments), thus we diluted the initial suspension so that $\phi_{\mathrm{PVA}}$ was brought down to $1 \mathrm{wt} \%$. Reproducibility of this procedure was confirmed by repeating the measurements at least three times for each of the three different samples at each $\mathrm{pH}$. The zeta potential of the silica/PVA suspensions was measured using an electrophoretic technique (Zetasizer nano zs, Malvern instruments Ltd, UK). To confirm that interparticle interactions are negligible during the measurements, we diluted the original suspension with deionized water and confirmed that results are independent of the concentration when diluting by a factor of up to 500 . The zero-shear viscosities $\eta_{0}$ were obtained from steady shear viscosities measured with an ARES strain-controlled rheometer (TA Instruments, Newark, USA) using a cone and plate geometry of $50 \mathrm{~mm}$ diameter and an angle of $0.0398 \mathrm{rad}$ at $25^{\circ} \mathrm{C}$.

Drying behavior The organization of silica particles during drying was observed with the vertical SAXS setup at the BW1 line at DESY (Hamburg, Germany), as schematically shown in Fig. 1. The silica/PVA mixture was loaded onto a horizontal VESPEL plate with a pipette and spread to form a circular film with a radius of $10 \mathrm{~mm}$. The vertical X-ray beam passed the sample at $5 \mathrm{~mm}$ from the center of the film. An extensive description on the setup, experiment and data treatment of SAXS intensity can be found in $\operatorname{ref}^{27}$. To assure homogeneity of the sample in the beam direction during drying, we performed experiments with increasing sample volumes from 0.1 to $0.3 \mathrm{ml}$ over the same area (corresponding to average film thicknesses of $0.3 \mathrm{~mm}$ to $1 \mathrm{~mm}$ ) and obtained reproducible and height independent scattering results. The surface microstructure of the dried film was separately determined using a scanning electron microscope (JSM-840A, JEOL, Japan). 


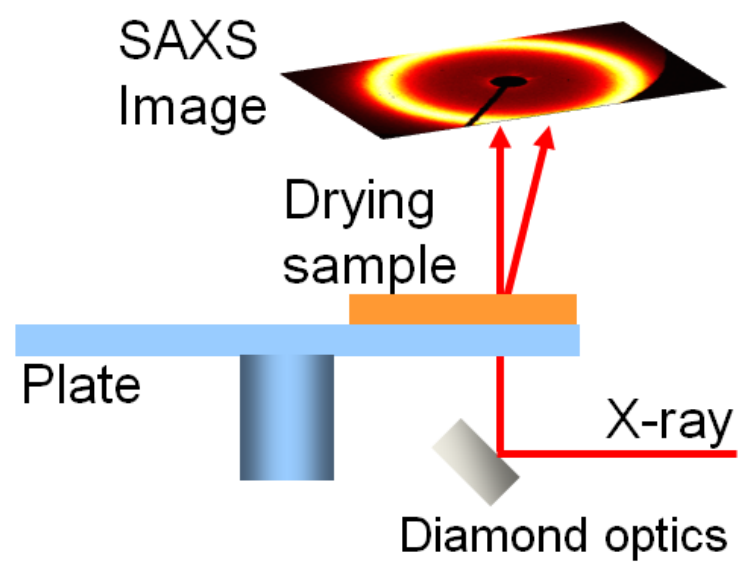

Figure 1. Sketch of a drying experiment with the vertical SAXS setup ${ }^{27}$.

\section{Results and discussion}

\subsection{Affinity between silica and PVA at different $\mathrm{pH}$ values, and dispersion state of NPs} in silica/PVA suspensions

In this section, we first evaluate the physicochemical affinity between the silica surface and PVA in the initial suspension at different $\mathrm{pH}$. It is well documented that, when the $\mathrm{pH}$ is reduced in silica/PVA suspensions, the silanol groups on silica surfaces are protonated (i.e. $\left.\mathrm{SiO}^{-}+\mathrm{H}^{+} \rightarrow \mathrm{SiOH}\right)$ and form hydrogen bonds with $\mathrm{PVA}^{29}$. Measuring the zeta potential $\zeta$ and the amount of PVA adsorption $\Gamma$ on the silica surface, it could be observed that $\zeta$ reduces and $\Gamma$ increases with lowering $\mathrm{pH}$ as shown in Fig. 2A, implying that silica surface is neutralized, likely as a result of the protonation of silanol group, and thus of the increased PVA adsorption. This suggests that the affinity between silica and PVA becomes more favorable with lowering $\mathrm{pH}$.

We now characterize the NP dispersion in the silica/PVA suspensions by means of SAXS intensity (Fig. 2B) and steady shear viscosity (Fig. 2C). At pH 10, a plateau in SAXS intensity is observed in the low q range, suggesting that the particles are stabilized as a result 
of the strongly negative $\zeta$ (Fig. 2A). When the $\mathrm{pH}$ is decreased, the intensity at low q starts to exhibit a negative slope, implying that the particles gradually lose their stability. Finally, at $\mathrm{pH} 3, \mathrm{I}(\mathrm{q})$ exhibits a power law slope, indicating that particles form a fractal-like flocculated structure. The zero shear viscosity $\left(\eta_{0}\right)$ significantly increases with decreasing $\mathrm{pH}$ (Fig. $2 \mathrm{C}$ ), also suggesting that the particles are flocculating.

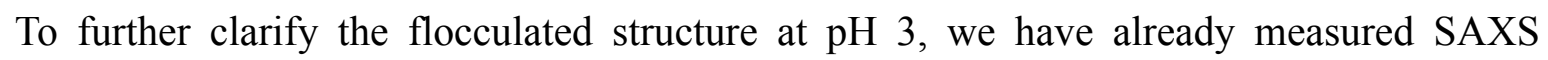
intensities and $\Gamma$ in an earlier publication when increasing the concentration $\phi_{\mathrm{PVA}}$ from 0 to 4 $\mathrm{wt} \%$, and have found that NP particles experience bridging flocculation followed by steric stabilization as $\Gamma$ increases up to $\phi_{\mathrm{PVA}}=2 \mathrm{wt}^{0} \%^{30}$. A further increase of $\phi_{\mathrm{PVA}}$ resulted in the NPs experiencing a depletion flocculation, where the cluster size increased when increasing $\phi_{\mathrm{PVA}}$ up to $4 \mathrm{wt}^{0} \%^{30}$. This suggested that silica particles at $\phi_{\mathrm{PVA}}=4 \mathrm{wt} \%$ form a depletion flocculated structure, where individual particles are sterically stabilized at short-range by adsorbed PVA. We are going to use this system and structure for the current drying study.
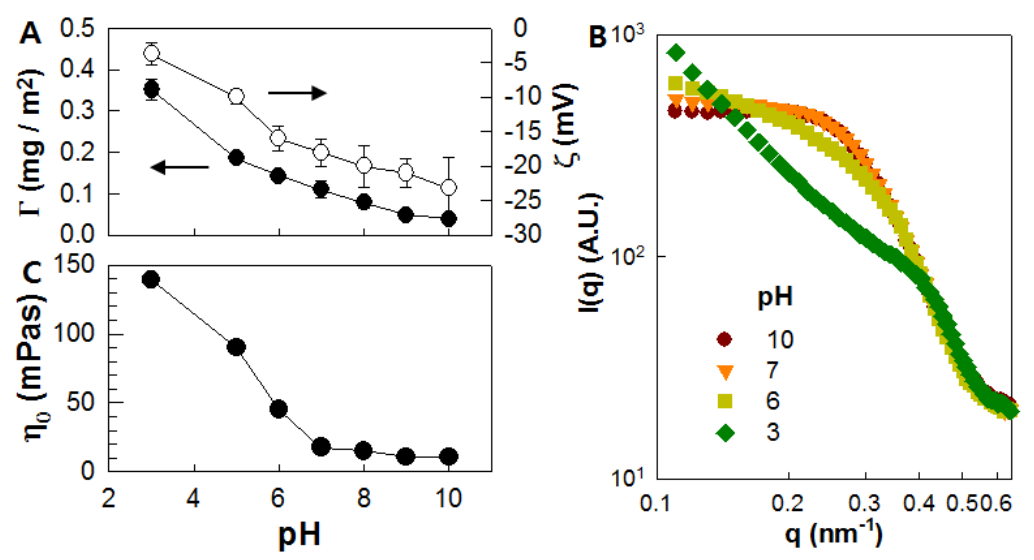

Figure 2. Surface chemistry of the silica surface and microstructure of silica/PVA suspension as a function of $\mathrm{pH}$. (A) Closed symbol: amount of polymer $\Gamma$ adsorbed onto the silica surface for a $1 \mathrm{wt} \%$ silica and $10 \mathrm{wt} \%$ PVA suspension. Open symbol: zeta potential $\zeta$ measured for the suspension of $4 \mathrm{wt} \%$ silica and $10 \mathrm{wt} \%$ PVA, diluted by a factor of $200-$ 
500. (B) SAXS intensity I(q) of the suspension (4 wt $\%$ silica and $10 \mathrm{wt} \%$ PVA) for he different $\mathrm{pH}$ values. (C) Zero shear viscosity, $\eta_{0}$ of the suspension (4 wt $\%$ silica and $10 \mathrm{wt} \%$ PVA).

\subsection{Structure of NP dispersion in the solid film after drying}

In this section, we investigate the structure of NP dispersion in the solid film after drying. Figs. 3A-C display images of the initially liquid silica/PVA films at different $\mathrm{pH}$ levels. The liquid films at pH 10 (Fig. 3A) and pH 7 (Fig. 3B) exhibit a transparent appearance as a result of charge-stabilization. On the other hand, at pH 3 (Fig. 3C) the liquid film exhibits a turbid image as a result of depletion attraction and a lack of electrostatic repulsion. Figs. 3D-F exhibit the images of the solid, dry films, which changed their transparency significantly compared to the liquid state (thus cannot be predicted from the transparency of the liquid state). At $\mathrm{pH} 10$ (Fig. 3D), the solid film exhibits a turbid appearance, with a porous microstructure consisting of dense aggregates (as seen from the SEM inset), suggesting that the charge-stabilized particles form aggregates during drying. On the other hand, the solid film at $\mathrm{pH} 7$ (Fig. 3E) exhibits a less turbidity than at $\mathrm{pH} 10$ and a disappearance of aggregates in the SEM image. In addition to this, we also observe a regular crack pattern at $\mathrm{pH} 7$, whereas no cracks were observable at $\mathrm{pH} 10$. Cracks occur when elastic stresses, caused by capillary pressure, exceed the strength of the material, resulting in a stress relaxation in form of cracks. The capillary pressure is proportional to $\gamma / r_{h}$ where $\gamma$ is the surface tension and $r_{h}$ is the pore radius, respectively. The less porous structure observed in the SEM images at $\mathrm{pH} 7$ suggests therefore a higher capillary pressure that results in the observed formation of cracks. At pH 3 (Fig. 3F), the initially turbid liquid film dries into a transparent solid film without observable aggregates in the SEM image. This implies that flocculate silica particles in the liquid state change their microstructure into a denser state 
during drying. The solid film at $\mathrm{pH} 3$ also exhibits a regular crack pattern that is also observed in drying films of stable suspensions as a result of close packed structure ${ }^{31,32}$. This suggests that NPs in the solid film at $\mathrm{pH} 3$ and $\mathrm{pH} 7$ form a dense structure, which results in a smaller pore size and introduces higher capillary pressures.
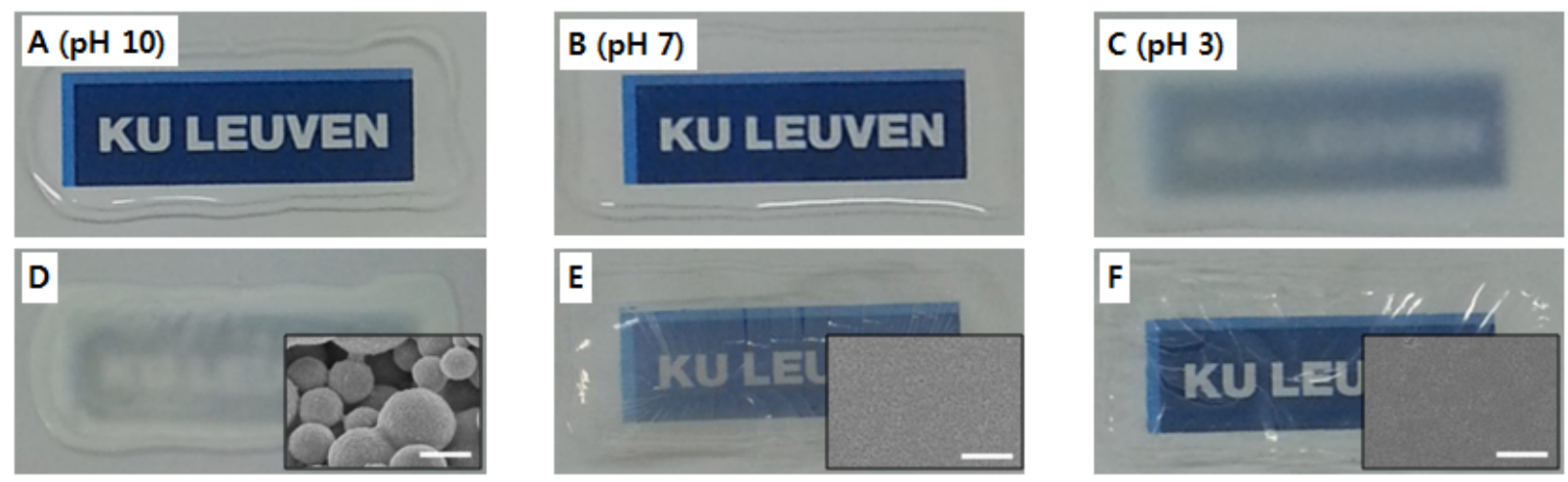

Figure 3. Silica/PVA film before and after drying at various pH. Visual image before (AC) and after (D-F) drying of the coating film casted onto a borosilicate slide glass. E and F exhibit cracks in the dried film that are, however, not visible in D. Size of the logo in the images is $40 \mathrm{~mm}$ (width) $\times 15 \mathrm{~mm}$ (height). Insets: SEM images of the dried films (the scale bar is $1 \mu \mathrm{m})$

To further understand the NP organization in the solid film, we measured SAXS intensities

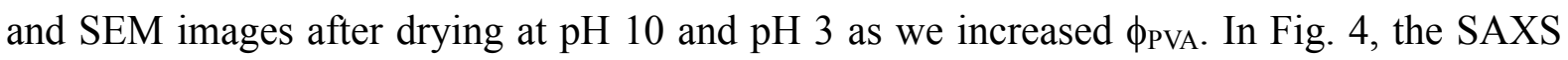
intensities at $\mathrm{pH} 10$ exhibit an upturn at the lowest $\mathrm{q}$ and a shoulder at $\mathrm{q}=0.43 \mathrm{~nm}^{-1}$, suggesting that particles form disordered aggregates. The shoulder at $\mathrm{q}=0.43 \mathrm{~nm}^{-1}$ allows us to approximate the center-to-center distance $d$ between particles as $d=2 \pi / \mathrm{q}=14.6 \mathrm{~nm}$, so nearly the same as the silica particle diameter. This implies that bare silica particles form aggregates in the polymer film, without any PVA in the aggregates. This also indicates that 
the spherical aggregates observed in the SEM image of (Figs. 4B-D) are surrounded by PVA, probably with surface tension shaping the silica aggregates when air intrudes the drying film at the late stage of drying. Interestingly, increasing the PVA concentration does not change SAXS intensity or peak position, but only fills the pore space between aggregates without changing their size and shape as can be seen in the SEM images (Figs. 4B to D). This suggests that PVA does not improve the particle dispersion at $\mathrm{pH} 10$ at all, due to the poor affinity between silica and PVA.
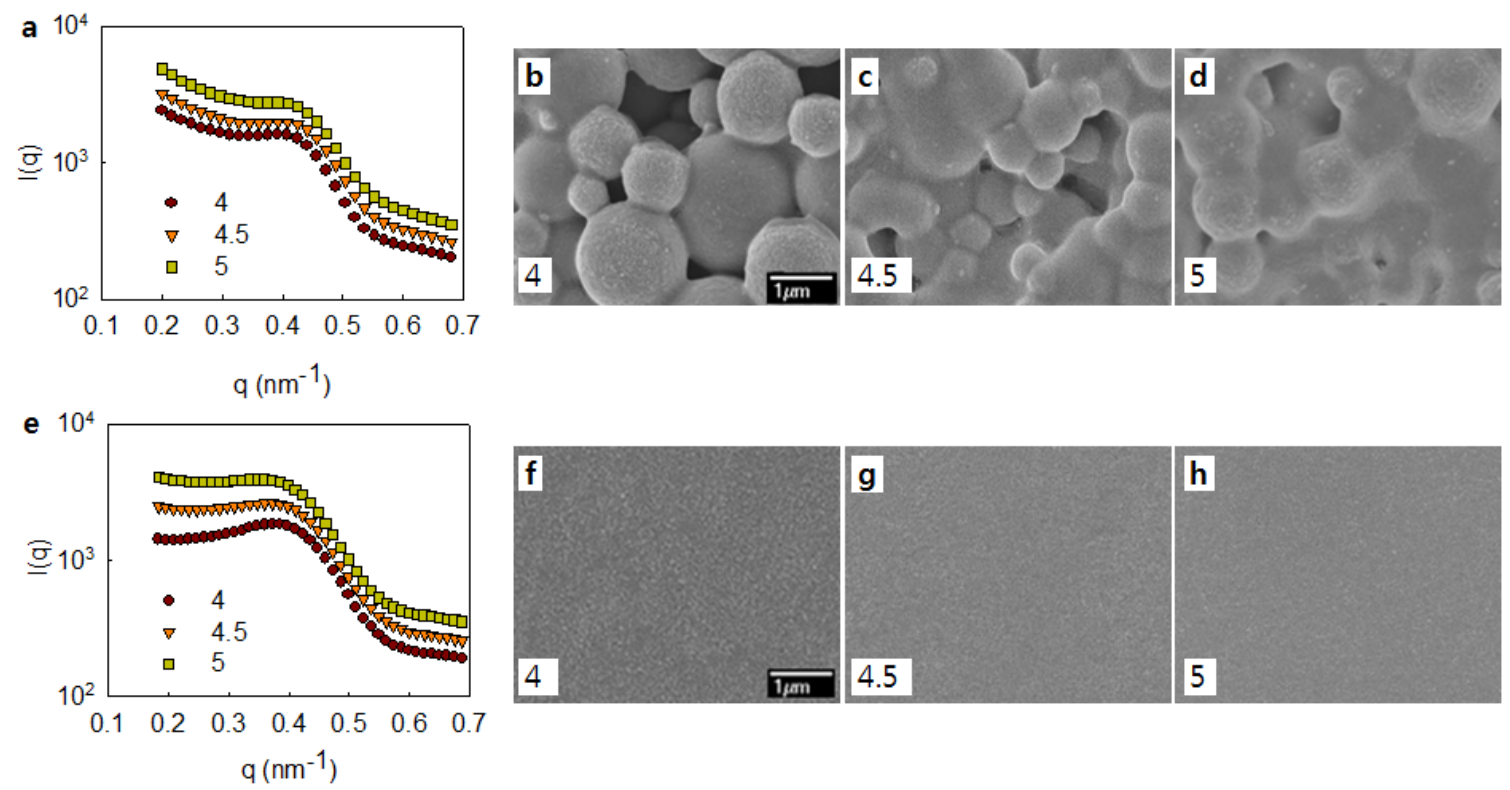

Figure 4. SAXS intensity and SEM images of solid film at pH10(a-d) and $\mathrm{pH} 3(\mathrm{e}-\mathrm{h})$ at different $\phi_{\mathrm{PVA}}$ of silica/PVA suspension. Films are dried from suspensions of $\phi_{\mathrm{PVA}}=4,4.5,5$ $\mathrm{wt} \%$ (as noted in the image), respectively, and a constant silica concentration of $10 \mathrm{wt} \%$. Volume ratio of the PVA and the silica in the film is $0.73,0.83$ and 0.92 for $\phi_{\mathrm{PVA}}=4,4.5$ and $5 \mathrm{wt} \%$, respectively.

In contrast to this, the SAXS intensity at $\mathrm{pH} 3$ and $\phi_{\mathrm{PVA}}=4 \mathrm{wt} \%$ (Fig. $\left.4 \mathrm{E}\right)$ exhibits a broad peak at $\mathrm{q}=0.3-0.4 \mathrm{~nm}^{-1}$ and a suppressed intensity at low $\mathrm{q}$. This indicates that particles are 
not aggregated, but rather form a dense and uniform structure (as supported by the regular crack pattern we mentioned in Fig. 3F). Furthermore, with increasing $\phi_{\mathrm{PVA}}$ the SAXS intensity shows a gradual disappearance of the broad peak at $\mathrm{q}=0.3-0.4 \mathrm{~nm}^{-1}$ and an upturn at the low q. This implies that the added PVA widens the distance among silica particles, but without changing the bulk structure, as shown in the SEM images (Figs. 4F-H).

The analysis of the solid structure in the silica/PVA film in comparison to the liquid state suggests that the structure of the NP dispersion strongly changes during drying. The affinity between particle and polymer seems to play a dominant role in the complex development of the NP dispersion during drying. To understand how the NP structure develops, we interpret the temporal evolution of vertical-SAXS intensities in the following section.

\subsection{Drying behavior investigated by vertical SAXS}

In this section, we examine how the SAXS intensity $\mathrm{I}(\mathrm{q})$ and structure factor $\mathrm{S}(\mathrm{q})$ change as solvent evaporates in the silica/PVA film at $\mathrm{pH} \mathrm{10,7}$ and 3, with the results shown in Figs. 5, 6 and 7, respectively. We note that, from an experimental point of view, the measured SAXS intensities of the suspensions exhibited a slight variation in the overall intensity for each sample loading, even for the same $\mathrm{pH}$, due to slight variations of thickness of the liquid film loaded on the plate in the SAXS setup. Furthermore, since we were not able to directly measure the thickness of initial film, it was not possible to scale I(q) with respect to the film thickness to correct for the intensity variation. Due to this experimental restraint, we ignore the absolute value of the vertical scale of I(q) in Figs. 5A, 6A and 7A, and arbitrarily shift I(q) in the vertical direction only for clarity issue. To obtain the structure factor $\mathrm{S}(\mathrm{q})$ within this limit for a given intensity, we combine the form factor $\mathrm{P}(\mathrm{q})$ with $\mathrm{I}(\mathrm{q})$ so that $\mathrm{S}(\mathrm{q})=\mathrm{I}(\mathrm{q}) / \mathrm{P}(\mathrm{q})$ $\sim 1$ at high $\mathrm{q}$, where $\mathrm{P}(\mathrm{q})$ was determined for the $0.1 \mathrm{wt} \%$ charge-screened silica suspension. 
Figs. 5A and 5B exhibit $\mathrm{I}(\mathrm{q})$ and $\mathrm{S}(\mathrm{q})$ of the suspension at $\mathrm{pH} 10$, respectively, with increasing $\tau$, where $\tau$ is the drying time normalized by the time at which the scattering intensity does not change anymore.
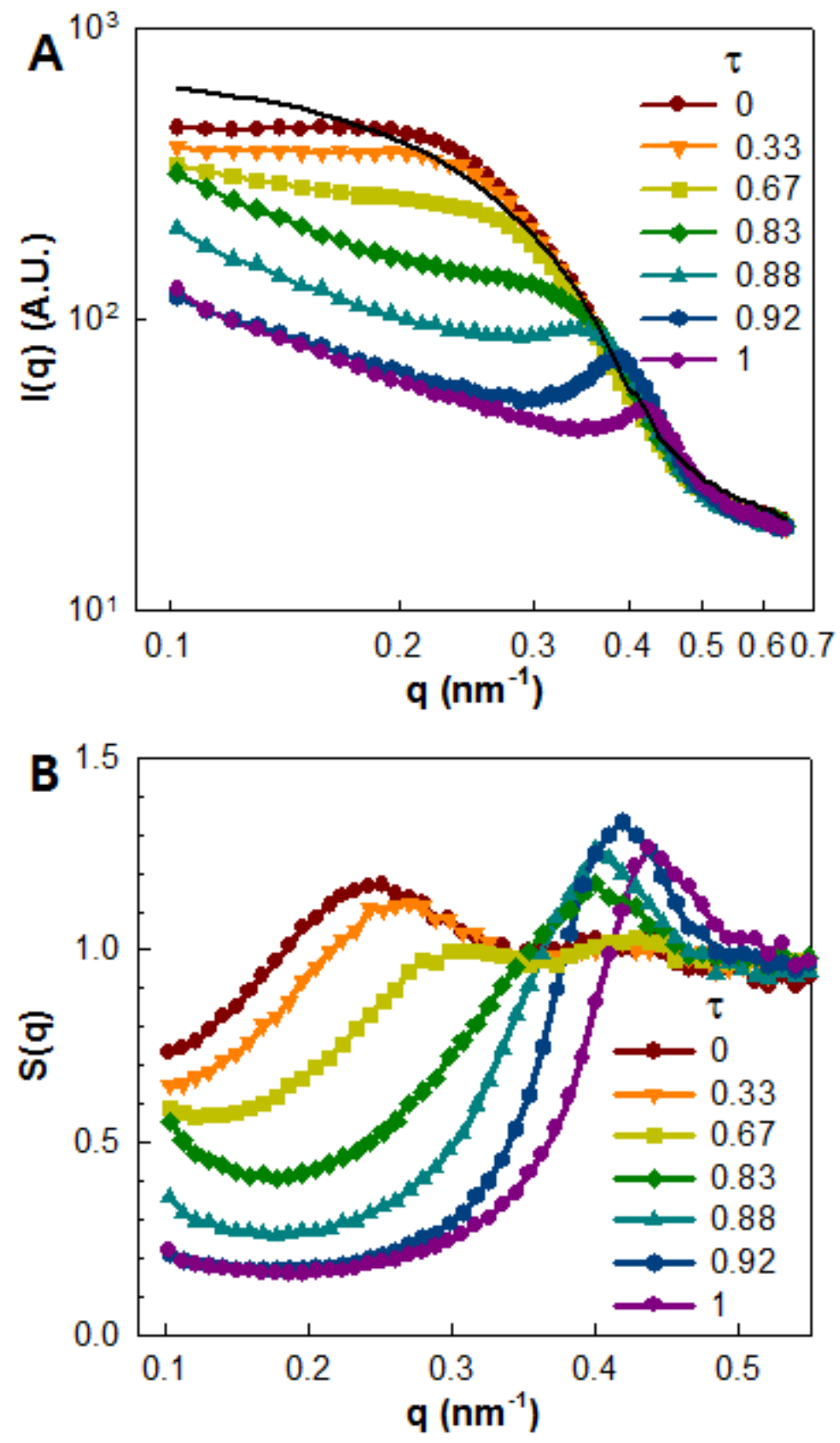

Figure 5. (A) SAXS intensity I(q) of the suspension of $4 \mathrm{wt} \%$ silica and $10 \mathrm{wt} \% \mathrm{PVA}$ at $\mathrm{pH}$ 10 with respect to the reduced drying time $\tau$. I(q) is vertically arbitrarily shifted for clarity. Solid line: form factor $\mathrm{P}(\mathrm{q})$, which is combined with $\mathrm{I}(\mathrm{q})$ so that $\mathrm{S}(\mathrm{q})=\mathrm{I}(\mathrm{q}) / \mathrm{P}(\mathrm{q}) \sim 1$ at high $\mathrm{q}$. 
(B) Structure factor $\mathrm{S}(\mathrm{q})$ for different $\tau$.

$\mathrm{S}(\mathrm{q})$ in Fig. 5B shows an intensity peak around $\mathrm{q}=0.25 \mathrm{~nm}^{-1}$, which gradually weakens and shifts to higher q during drying, and finally disappears at $\tau=0.67$. This indicates that the charge-stabilized particles are gradually losing their long-range stability. At $\tau=0.67$ a slight upturn is observed at the lowest q, suggesting that particles at this point start to weakly aggregate (see schematics in Fig. 8A at $\tau=0.67$ ). In our previous drying study of identical charged-particles in the absence of $\mathrm{PVA}^{27}$, we show that, when solvent evaporates, the upconcentrating sodium ions in the medium weaken the electrostatic repulsion as they reduce the distance over which electrostatic effects persist (i.e. the Debye length ${ }^{33}$ ). The same will happen also in the present system, thus reducing electrostatic repulsion. In addition, the increasing concentration of PVA in the medium also contributes to the observed destabilization. This is because the amount of PVA that can be adsorbed is limited and set by the surface area of silica. Therefore, the concentration of non-adsorbed PVA in the medium is expected to increase as solvent evaporates, suggesting that depletion attraction may become stronger during drying, which possibly contributes to the observed destabilization in the drying film. In fact, the gradual disappearance of peak intensity at $\mathrm{q}=0.25 \mathrm{~nm}^{-1}$ during drying shows a marked difference from our previous drying experiment in the absence of $\mathrm{PVA}^{27}$, which exhibited a stronger peak intensity, followed by an abrupt disappearance at the near-end of drying. This suggests that depletion attraction plays an important role already from the early stage of drying as a driving force that leads to the destabilization in the following drying stage.

At $\tau=0.83$, S(q) develops a pronounced peak at $\mathrm{q}=0.4 \mathrm{~nm}^{-1}$ and a significant upturn at the lowest $\mathrm{q}$, indicating that the destabilized NPs form aggregates. This peak at $\mathrm{q}=0.4 \mathrm{~nm}^{-1}$ 
shifts to $0.43 \mathrm{~nm}^{-1}$ over the time span $0.83<\tau<1$. The final value of $\mathrm{q}=0.43 \mathrm{~nm}^{-1}$ is equivalent to the particle size (as discussed in Fig. 4A). This suggests that over this stage the increasing concentration of non-adsorbed polymers introduces a strong depletion attraction, which eventually leads to a separation into a phase of bare particles and a polymer phase. At $\tau$ $=0.92 \sim 1$, we observe an increase in the scattering contrast (see Supporting Information Fig. $\mathrm{S} 1$ ), indicating that air intrudes into the film ${ }^{27}$, which results in a turbid film after drying (Fig 3D).

Based on the analysis of $\mathrm{S}(\mathrm{q})$, we suggest the following scheme of NP dispersion evolution in the silica/PVA suspension during drying as illustrated in Fig. 8A: The suspension shows dispersed NPs at $\tau=0$ that gradually lose stability at $\tau=0.33$, followed by the occurance of disordered NP aggregates at $\tau=0.67$. During the further drying process, the suspension contains strong aggregate of bare NPs with a direct contact of the bare surfaces, resulting in a separation of the polymer phase and the particle phase from $\tau=0.83$ to 0.92 , consecutively. Finally, NP aggregates remain in the solid film after air intrudes inbetween the aggregates at $\tau$ $=1$. 

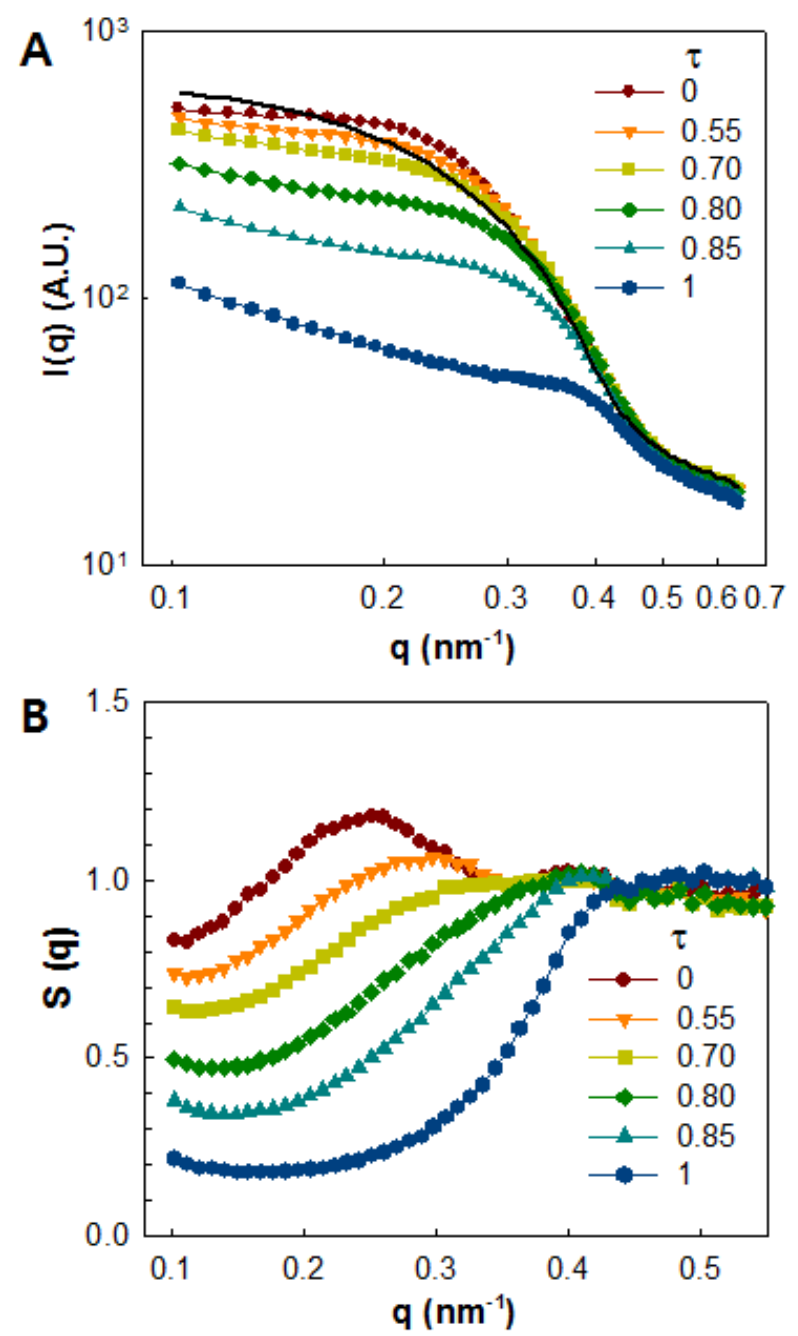

Figure 6. (A) SAXS intensity I(q) of the suspension of $4 \mathrm{wt} \%$ silica and $10 \mathrm{wt} \% \mathrm{PVA}$ at $\mathrm{pH} 7$ for different reduced drying times $\tau$. I(q) is vertically shifted for clarity issue. Solid line: form factor $\mathrm{P}(\mathrm{q})$, which is combined with $\mathrm{I}(\mathrm{q})$ so that $\mathrm{S}(\mathrm{q})=\mathrm{I}(\mathrm{q}) / \mathrm{P}(\mathrm{q}) \sim 1$ at high $\mathrm{q}$. (B) Structure factor $S(q)$ with respect to $\tau$.

The evolution of $\mathrm{I}(\mathrm{q})$ and $\mathrm{S}(\mathrm{q})$ of the drying suspension at $\mathrm{pH} 7$ is displayed in Fig. 6A and $6 \mathrm{~B}$ respectively. The evolution of $\mathrm{S}(\mathrm{q})$ from $\tau=0$ to 0.7 shows a behavior similar to $\mathrm{pH} 10$, indicating that also in this case silica particles gradually lose their long-range stability as the solvent evaporates, possibly due to the increasing depletion attraction. However, when comparing to $\mathrm{pH} 10$ (Fig. 5B) for the time $0.83<\tau<1$, S(q) at $\mathrm{pH} 7$ for $0.8<\tau<1$ (Fig. 6B) 
shows neither a significant upturn at the lowest q, nor the intensity peak at high q, suggesting that the silica/PVA film dries without phase separation, which leads to an improved transparency of the solid film at pH 7 (Fig. 3E) compared to pH 10 (Fig. 3D). However, this absence of strong aggregation and phase separation is on the first glance counterintuitive as charge stabilization at $\mathrm{pH} 7$ is lower than at $\mathrm{pH} 10$. This points at the important role that the polymer plays in this context. Compared to $\mathrm{pH} 10$, the silica/PVA suspension at $\mathrm{pH} 7$ has a slightly higher amount of polymer adsorption as seen in Fig 2. Studies on depletion attraction in the presence of grafting polymers ${ }^{34-36}$ showed that the presence of grafting polymer improves the dispersion stability, not only by introducing steric repulsion, but also by suppressing depletion attraction due to interpenetration of the free polymer into the steric layer of (anchored) polymer chains around the particles. Based on these studies, the improved NP dispersion in the solid film is likely to be caused by the increased PVA adsorption, which introduces steric repulsion and suppresses the depletion attraction during drying. 

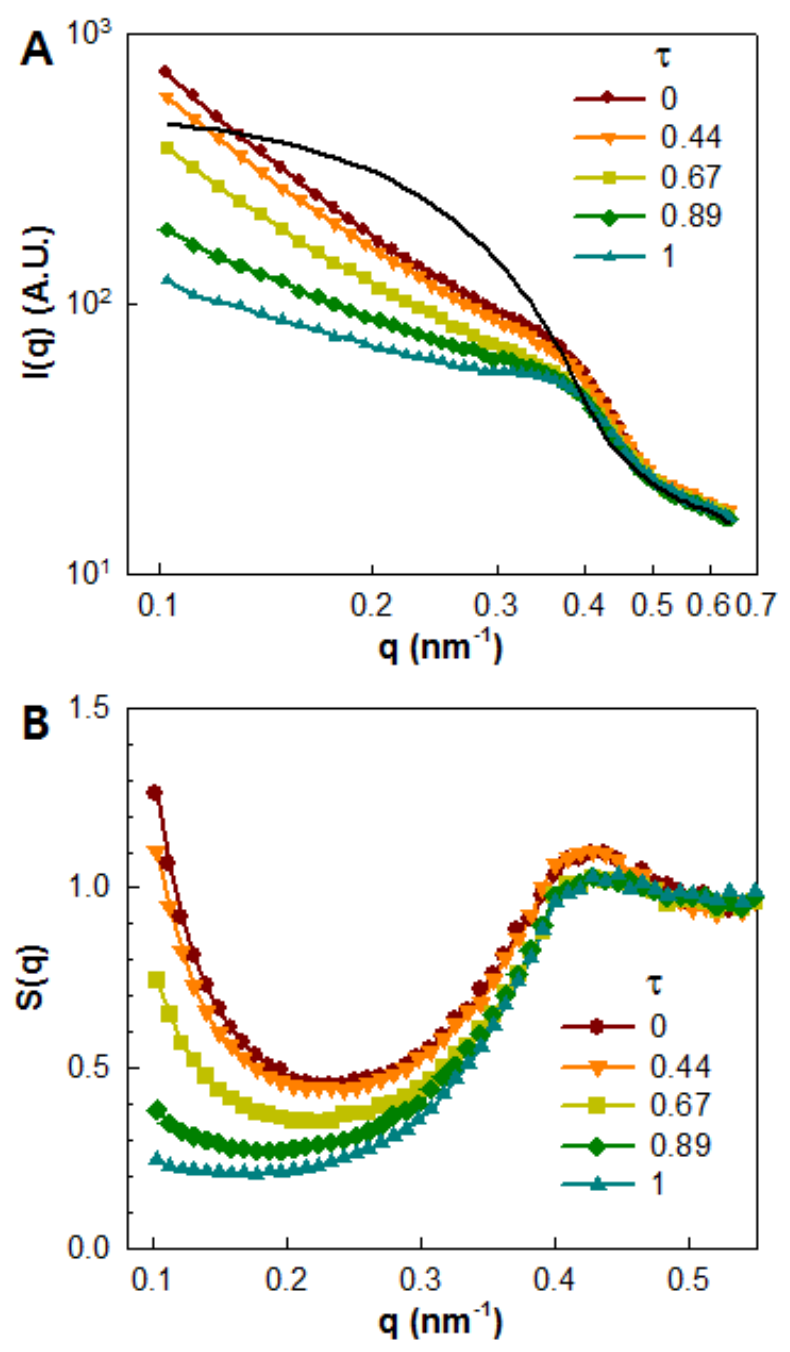

Figure 7. (A) SAXS intensity I(q) of the suspension of $4 \mathrm{wt} \%$ silica and $10 \mathrm{wt} \% \mathrm{PVA}$ at $\mathrm{pH} 3$ with respect to the reduced drying time $\tau$. I(q) is vertically shifted for clarity issue. Solid line: form factor $\mathrm{P}(\mathrm{q})$ which is combined with $\mathrm{I}(\mathrm{q})$ so that $\mathrm{S}(\mathrm{q})=\mathrm{I}(\mathrm{q}) / \mathrm{P}(\mathrm{q}) \sim 1$ at high $\mathrm{q}$. (B) Structure factor $S(q)$ with respect to $\tau$.

The evolution of $\mathrm{I}(\mathrm{q})$ and $\mathrm{S}(\mathrm{q})$ during drying at $\mathrm{pH} 3$ is displayed in Figs. $7 \mathrm{~A}$ and $7 \mathrm{~B}$, respectively. $\mathrm{S}(\mathrm{q})$ shows a dramatic reduction of the upturn at the lowest $\mathrm{q}$, which indicates that silica particles change their microstructure from depletion clusters to a uniform distribution as their volume fraction increases during drying. However, the intensity in the 
high $\mathrm{q}$ range does not change, indicating that the distance between neighboring silica particles stays nearly the same throughout the drying process. This suggests a simple picture of the structural development during drying as illustrated in Fig. 8B: the initial aggregates are simply concentrating, with the particles eventually changing their relative positions within the aggregates to accommodate into the final close packing in the dry film (but keeping a constant inter-particle distance during the reorganization). The adsorbed polymers on the silica surface play a key role in the observed structural evolution as they sterically stabilize the particles on the short-range during all the drying process and thus enable the close range reorganization of particles.
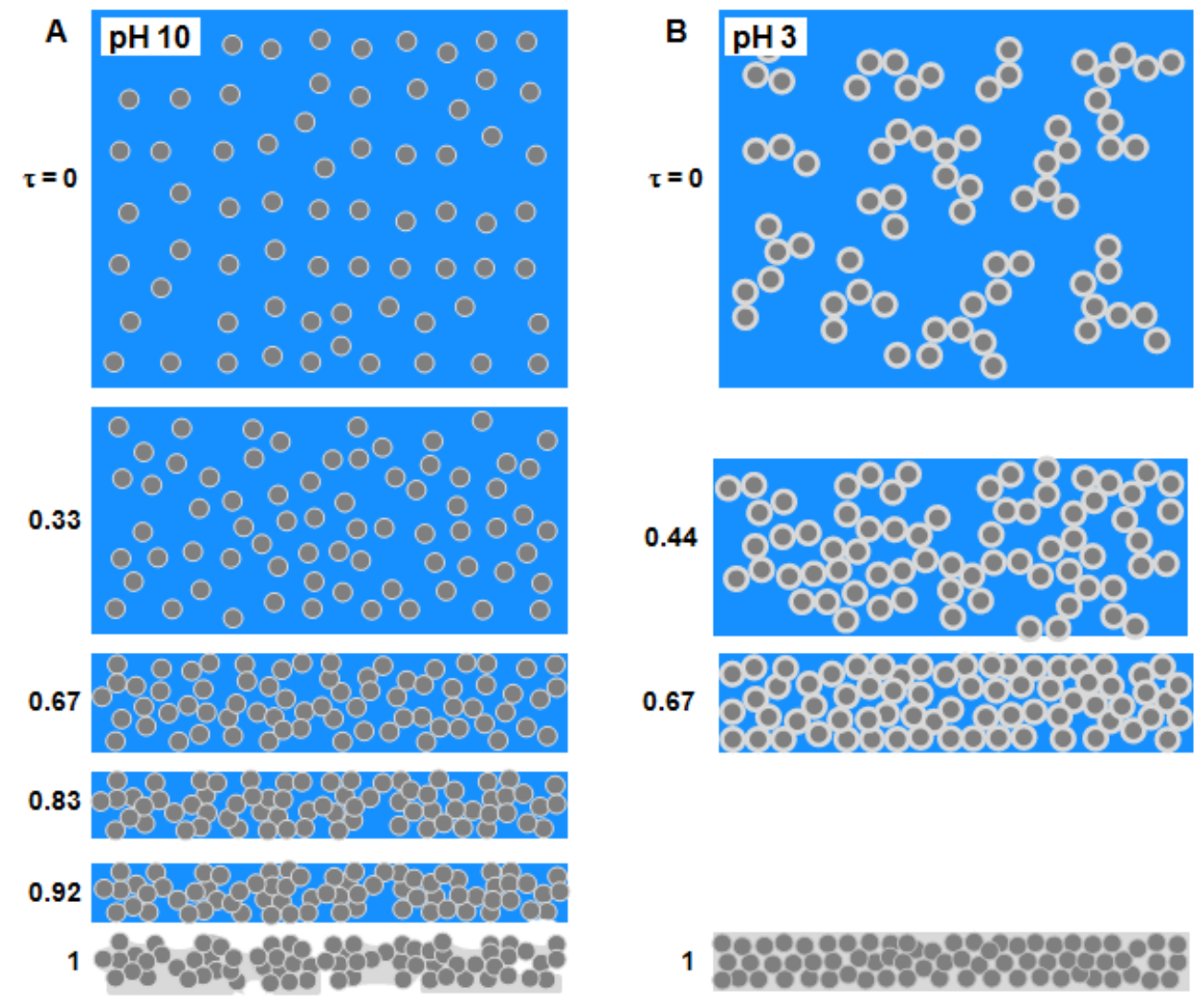
Figure 8. Schematic of the structural evolution of silica dispersions during the drying of silica/PVA films at (A) pH 10 and (B) pH 3. Reduced values of film thickness are estimated from solvent loss measurements (See Supporting Information S2)

In order to further understand the evolution of SAXS intensity and corresponding NP dispersion during drying, we quantify $\mathrm{I}(\mathrm{q})$ and the $\mathrm{S}(\mathrm{q})$ as a function of $\tau$. The intensity in the low q range of I(q) reflects the long-range structure of NPs (for example, a power law slope at low q reflects the fractal dimension of NP clusters ${ }^{30}$ ). Therefore, we determine the absolute value of the power law slope $\alpha$ from I(q) for each different $\mathrm{pH}$ of the suspensions (Figs. 5A, 6A and 7A) over the range $0.1 \mathrm{~nm}^{-1}<\mathrm{q}<0.2 \mathrm{~nm}^{-1}$, with the results displayed in Fig. 9. The $\alpha$ value for $\mathrm{pH} 10$ is initially zero, implying stabilized NPs in the suspension. With increasing $\tau$, $\alpha$ rises gradually up to 1 , implying that stabilized NPs are destabilized and then form NP clusters during drying as schematically depicted in Fig. 8A. For $\mathrm{pH} 7$ the value of $\alpha$ is 0.2 at the early stage of drying, indicating that NPs at pH 7 are initially less stabilized than at $\mathrm{pH} 10$. However, $\alpha$ rises during drying only up to 0.8 , which is smaller than for $\mathrm{pH} 10$, implying that cluster formation during drying at $\mathrm{pH} 7$ is less significant. The suspension at $\mathrm{pH} 3$ shows $\alpha=$ 2.1 in the beginning, indicating a significant initial presense of NP clusters in the suspension. However, $\alpha$ gradually decreases during drying down to the level observed for $\mathrm{pH}$ 7. This implies that the NP clusters disappears during drying with increasing concentrating, as schematically depicted in Fig. 8B. 


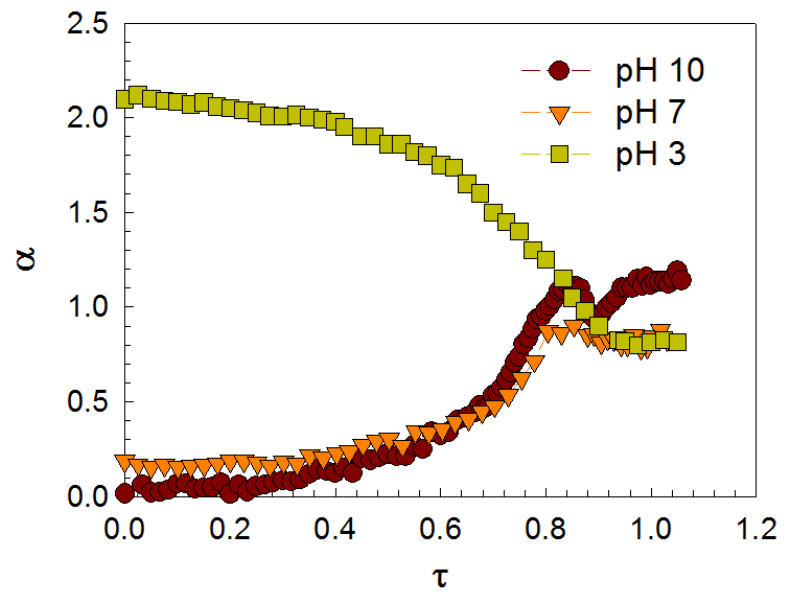

Figure 9. Absolute value of the power law slope $\alpha$ as a function of $\tau$, obtained from I(q) of Figs. 5a, 6a and 7a over the range $0.1 \mathrm{~nm}^{-1}<\mathrm{q}<0.2 \mathrm{~nm}^{-1}$. All three suspensions are composed of $4 \mathrm{wt} \%$ silica and $10 \mathrm{wt} \%$ PVA.

Information about the arrangement of NPs over short ranges are mainly obtained from analyzing the primary peak in $\mathrm{S}(\mathrm{q})$. For example, the average inter-particle distance and the degree of NP arrangement are obtained from the position and intensity of the primary peak in $S(q)$, respectively ${ }^{27}$. Here we quantify the evolution of $S(q)$ during drying by tracking the position of the primary peak $\mathrm{q}_{\text {peak }}$ and the peak intensity $\mathrm{S}_{\text {peak }}$ as a function of $\tau$, with results shown in Figs. 10A and 10B, respectively.

The $\mathrm{q}_{\text {peak }}$ data of $\mathrm{pH} 10$ and $\mathrm{pH} 7$ show a very similar time dependency in Fig. 10A; a gradual increase during drying, implying that the average interparticle distance reduces during drying in a similar way. However, Fig. 10B exhibits a marked difference in the evolution of $\mathrm{S}_{\text {peak }}$ for $\mathrm{pH} 10$ and $\mathrm{pH}$ 7, in particularly from $\tau=0.8$ onwards. A sudden rise of $\mathrm{S}_{\text {peak }}$ for $\mathrm{pH} 10$ indicates the formation of dense aggregates of NPs, whereas no rise is observed for $\mathrm{pH} \mathrm{7,} \mathrm{indicating} \mathrm{that} \mathrm{no} \mathrm{NP} \mathrm{aggregates} \mathrm{form.} \mathrm{It} \mathrm{is} \mathrm{notable} \mathrm{that} \mathrm{this} \mathrm{difference}$ between the two $\mathrm{pH}$ occurs only at the late stage of drying, possibly when NPs are getting so 
close that adsorbed polymers can mediate the NP arrangement. This again suggests that the adsorbed polymer plays a critical role in NP dispersion during drying.

It should be noted that $\mathrm{q}_{\text {peak }}$ for $\mathrm{pH} 3$ (Fig. 10A) shows a nearly constant value over the whole range of $\tau$, indicating that the particle arrangement remains constant throughout the drying process. The value $\mathrm{q}_{\text {peak }}=0.43 \mathrm{~nm}^{-1}$ gives the averaged interparticle distance of NPs from $2 \pi / \mathrm{q}$, yielding $14.6 \mathrm{~nm}$. This is equivalent to the diameter of silica particle, suggesting that NPs are touching the neighboring particles already from the beginning to the end of drying. On the other hand, $\mathrm{S}_{\text {peak }}$ in Fig. 10B gradually reduces during drying, indicating that the initially heterogeneous structure of aggregated NP clusters gradually compacts into a uniform distribution. The constant $\mathrm{q}_{\mathrm{peak}}$ and the reduction of $\mathrm{S}_{\text {peak }}$ during drying at $\mathrm{pH} 3$ suggest that the isolated NP aggregates are uniformly distributed during drying while maintaining their direct connection to neighboring NPs. We have noted in section 3.1 and also in ref 30 that the NPs in suspension at $\mathrm{pH} 3$ are depletion flocculated at long-range, but sterically stabilized by adsorbed polymer at short-range. Thus it is expected that individual NPs are still allowed to change their relative position, even at close contact, with the help of the short-range steric repulsion, while they sustain their direct touching status due to the longrange depletion attraction. This leads then to the uniform distribution, as schematically shown in Fig. 8B. Again, this suggests that the adsorbed polymer plays a critical role in the stabilization of NPs during drying. 

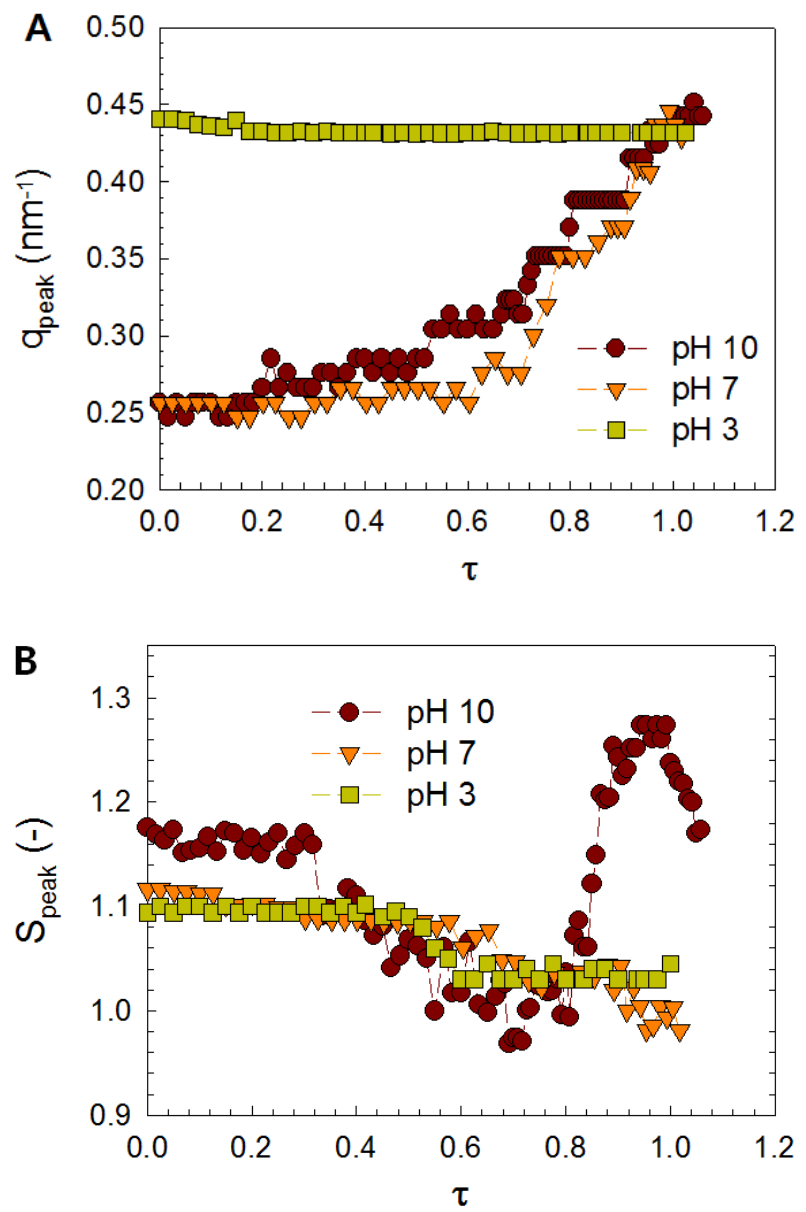

Figure 10. Quantification of S(q) (obtained from Figs. 5b, 6b and 7b) as a function of $\tau$. (A) Position of the primary peak $\mathrm{q}_{\text {peak }}$ of $\mathrm{S}(\mathrm{q})$ and (B) intensity $\mathrm{S}_{\text {peak }}$ of this peak. The composition of all three suspensions is $4 \mathrm{wt} \%$ silica and $10 \mathrm{wt} \%$ PVA.

\section{Discussion: The role of polymers in particle organization during drying}

The analysis of SAXS intensity presented above on the drying film suggests that the evaporation of solvent significantly changes the particle interaction in the silica/PVA films in a way that could not be predicted a priori from the initial solution properties. The increasing volume fraction of silica particle and non-adsorbed polymer due to solvent evaporation contributes strongly to the evolution of particle interaction. In addition to this, also the 
increasing salt concentration in the medium can reduce electrostatic repulsion ${ }^{27}$. To elucidate and quantify the so far qualitatively assumed effect of solvent evaporation on the particle interaction, we calculate in the following the particle interaction potentials and their evolution; depletion interaction $\left(\mathrm{V}_{\text {dep }}\right)$, steric repulsion $\left(\mathrm{V}_{\mathrm{s}}\right)$, electrostatic repulsion $\left(\mathrm{V}_{\mathrm{e}}\right)$ and van der Waals attraction $\left(\mathrm{V}_{\mathrm{vdw}}\right)$. To consider the effect of solvent evaporation on particle interaction, we assume that solvent evaporation increases the concentration of non-adsorbed PVA and ionic strength ${ }^{27}$, but does not affect the amount of adsorbed PVA and the Hamaker constants. Therefore, solvent evaporation is assumed to affect only $V_{\text {dep }}$ and $V_{e}$, without changing $V_{s}$ and $\mathrm{V}_{\mathrm{vdw}}$. On the basis of these assumptions, we compare the particle interaction potentials at different $\mathrm{pH}$ values for the initial state ( 0 vol\% solvent evaporation) with a representative intermediate state of $50 \mathrm{vol} \%$ solvent evaporation.

Steric repulsion Vs We employ scaling theory to determine the steric repulsion potential $\mathrm{V}_{\mathrm{s}}, 37,38$

$$
V_{S}=\pi(r+\delta) K \Phi_{s 0}^{9 / 4} D_{S c}\left\{-16 \Pi D_{S c} \ln \left(\frac{2 \delta}{h-2 r}\right)+\frac{4 D_{S c}^{5 / 4}}{2^{5 / 4}}(8 \Pi)^{9 / 4}\left[\frac{1}{(h-2 r)^{1 / 4}}-\frac{1}{(2 \delta)^{1 / 4}}\right]\right\}
$$

where $r$ and $\delta$ are the particle radius and adsorption layer thickness, respectively. $D_{\text {sc }}$ is the effective thickness of the adsorbed polymer. $\Pi$ and $\Phi_{\mathrm{s} 0}$ are the adsorption coverage $\left(\Pi=\Gamma / \Gamma_{0}\right)$ and the volume fraction of an adsorbed layer at the saturation, respectively. To determine the parameters and calculate $\mathrm{V}_{\mathrm{s}}$ at each $\mathrm{pH}$, we employ the following assumptions about the adsorbed layer. Firstly, we assume that the volume fraction of the polymer in the adsorption layer is uniform and independent of the distance from surface. According to this 
assumption $D_{\mathrm{sc}}=\delta$. Secondly, based on a previous study ${ }^{30}, \Pi=0.8$ at $\phi_{\mathrm{PVA}}=4 \mathrm{wt} \%$ at $\mathrm{pH} 3$, and we assume $\Pi=0.8$ for all $\mathrm{pH}$. Thirdly, we set $\Phi_{\mathrm{s} 0}=0.18$ at $\mathrm{pH} 3$ according to ref. ${ }^{37}$ and assume a decrease proportional to the measured $\Gamma$ with increasing $\mathrm{pH}$. As a result, we can calculate the adsorbed layer thickness from the amount of adsorbed polymer via $\delta=\Gamma /\left(\sigma \phi_{s 0} \Pi\right) . K$ is a constant expressed by $K=\left(\alpha_{S c} / a_{m}^{3}\right) k_{B} T$ where $\alpha_{\text {sc }}$ and $a_{m}^{3}$ are numerical constants that are proportional to the osmotic pressure and the segment length of a polymer chain, respectively ${ }^{37}$. For this calculation, we use $\mathrm{K}=3 \times 10^{5} \mathrm{~N} / \mathrm{m}^{2}$ at all $\mathrm{pH}$ levels following $\operatorname{ref}^{37}$, resulting in a magnitude of $\mathrm{V}_{\mathrm{s}}$ that is comparable to the other potentials. The parameter values for the calculation of $\mathrm{V}_{\mathrm{s}}$ used in Eq. (1) are summarized in Table 1.

\begin{tabular}{cccc}
\hline $\mathbf{p H}$ & $\mathbf{1 0}$ & $\mathbf{7}$ & $\mathbf{3}$ \\
\hline$\Gamma\left(\mathbf{m g} / \mathbf{m}^{\mathbf{2}}\right)$ & 0.03 & 0.14 & 0.36 \\
$\boldsymbol{\Phi}_{\mathrm{s} 0}$ & 0.05 & 0.1 & 0.18 \\
$\delta(\mathbf{n m})$ & 0.62 & 1.4 & 2.0 \\
\hline
\end{tabular}

Table 1. Parameter values for the calculation of $\mathrm{V}_{\mathrm{s}}$, Eq (1).

Depletion attraction $\boldsymbol{V}_{\text {dep }}$ The current system includes both adsorbing and free polymers, thus an estimation of the interpenetration of the matrix polymer molecules with the adsorbed polymer needs to be done when evaluating depletion attraction. Vincent and coworkers ${ }^{35,39}$ proposed a modified version of the classical Asakura-Oosawa model ${ }^{40}$ considering an interaction between adsorbing and free polymer. In this study, we employ the $V_{\text {dep }}$ of Vincent et al., which is given by $^{35}$ 


$$
V_{d e p}=-2 \pi r P_{2}\left(\Delta+\delta-p-\frac{h-2 r}{2}\right)^{2}
$$

where $P_{2}$ is the osmotic pressure of the bulk polymer solution, which dominantly depends on the bulk polymer concentration. $P_{2}$ is given by

$$
P_{2}=-\left(\frac{\mu_{1}-\mu_{1}^{0}}{v_{1}}\right)=\frac{k T}{v_{1}}\left[\ln \left(1-\phi_{2}^{b}\right)+\phi_{2}^{b}+\chi\left(\phi_{2}^{b}\right)^{2}\right]
$$

where $\chi$ is the Flory-Huggins parameter $\left(=0.46\right.$ for PVA in water $\left.{ }^{45}\right)$ and $v_{1}$ is the molar volume of the solvent. $\Delta$ in Eq. (2) is the range affected by depletion attraction, which can be approximated as the diameter of the polymer coil, given by ${ }^{35}$

$$
\Delta=1.4 r_{g}\left(\frac{1-\phi_{2}^{b}}{1-\phi_{2}^{*}}\right)
$$

where $r_{g}, \phi_{2}^{b}$ and $\phi_{2}^{*}$ are the radius of gyration, bulk concentration and critical overlap concentration of free polymer, respectively. In this study, we determined $\phi_{2}^{*}=1.4 \mathrm{wt} \%$. $p$ in Eq. (2) is the interpenetration distance of the matrix polymer chain into the adsorbed layer, reflecting the degree of interaction between adsorbed and bulk polymer. $p$ is given by ${ }^{35}$

$$
p=\frac{P_{2} \Delta v_{1}}{k T(1-2 \chi) \bar{\phi}_{2}^{a} \phi_{2}^{b}}
$$

where $\bar{\phi}_{2}^{a}$ is the volume fraction of adsorption layer, which is equal to $\phi_{\mathrm{s} 0} \Pi \cdot p$ reflects the degree of interaction between adsorbed and bulk polymer, thus depends not only on the bulk and adsorbed polymer concentration but also on the thickness of the adsorbed layer ${ }^{35}$. Since these values change during drying, the parameters calculated for conditions of 0 and $50 \mathrm{wt} \%$ solvent evaporation, respectively, are summarized in Table 2 


\begin{tabular}{cccc}
\hline $\mathbf{p H}$ & $\mathbf{1 0}$ & $\mathbf{7}$ & $\mathbf{3}$ \\
\hline$\phi_{\mathbf{n a}}(\mathbf{v o l} \%)$ & $3.53 / 6.58^{*}$ & $3.28 / 6.34^{*}$ & $2.79 / 5.85^{*}$ \\
$\mathbf{P}_{\mathbf{2}}\left(\times \mathbf{1 0}^{\mathbf{3}} \mathbf{P a}\right)$ & $5.76 / 25.65^{*}$ & $4.62 / 23.32^{*}$ & $3.16 / 19.01^{*}$ \\
$\Delta \mathbf{( n m})$ & $16.55 / 16.01^{*}$ & $16.56 / 16.03^{*}$ & $16.64 / 16.10^{*}$ \\
$\boldsymbol{p}(\mathbf{n m})$ & $0.60 / 0.61^{*}$ & $0.95 / 1.28^{*}$ & $0.90 / 1.21^{*}$ \\
\hline
\end{tabular}

Table 2. Parameter values for the calculation of $V_{\text {dep }}$ from Eq. (2). *values used for the calculation after evaporation of $50 \%$ of the solvent from the suspensions.

Electrostatic repulsion $\mathbf{V}_{\mathbf{e}}$ The Electrostatic repulsion potential $\left(\mathrm{V}_{\mathrm{e}}\right)$ is given by ${ }^{41}$

$$
V_{e}(h)=4 \pi \varepsilon \varepsilon_{0} r^{2} \psi_{0} \exp [-\kappa(h-2 r)] / h
$$

where $\psi_{0}$, and $\kappa$ are the surface potential and the Debye Hückel inverse screening length, respectively. $\varepsilon$ and $\varepsilon_{0}$ are the relative permittivity and the permittivity of vacuum, respectively. Our previous study ${ }^{27}$ on drying of charged silica has shown that the solvent evaporation increases ion concentration, and thus reduces $\psi_{0}$ and $\kappa^{-1}$, resulting eventually in a weakening of $\mathrm{V}_{\mathrm{e}}$. The correlation of the solvent concentration with $\psi_{0}$ and $\kappa^{-1}$ was derived as $\psi_{0} \sim \kappa^{-1} \sim$ $\sqrt{\left(1-\phi_{s}\right) / \phi_{s}}{ }^{27}$, where $\phi_{\mathrm{s}}$ is volume fraction of the solvent. This allows us to determine $\psi_{0}$ and $\kappa^{-1}$ both at 0 and $50 \mathrm{wt} \%$ solvent evaporation, respectively, as summarized in Table 3.

\begin{tabular}{cccc}
\hline $\mathbf{p H}$ & $\mathbf{1 0}$ & $\mathbf{7}$ & $\mathbf{3}$ \\
\hline$\psi_{\mathbf{0}}(\mathbf{m V})$ & $45 / 24^{*}$ & $35 / 21^{*}$ & $5 / 3^{*}$ \\
\hline
\end{tabular}


Table 3. Parameter values for the calculation of $\mathrm{V}_{\mathrm{e}}$ from Eq. (6). ${ }^{*}$ values used for the calculation for conditions after evaporation of $50 \%$ of the solvent from the suspension.

Van der Walls attraction $\mathbf{V}_{\mathbf{v d w}}$ The adsorbed layer on the particles can affect the Van der Waals attraction potential between particles. Therefore, we calculate $\mathrm{V}_{\mathrm{vdw}}$ in the presence of such an adsorbed layer, which is given by ${ }^{42}$

$$
\begin{gathered}
V_{v d w}(h)=-\frac{1}{12}\left\{\left(\sqrt{A_{22}}-\sqrt{A_{33}}\right)^{2} \frac{r+\delta}{h-2 r}+\left(\sqrt{A_{33}}-\sqrt{A_{11}}\right)^{2} \frac{r}{h-2 r+2 \delta}\right. \\
\left.+4 r\left(\sqrt{A_{22}}-\sqrt{A_{33}}\right)\left(\sqrt{A_{33}}-\sqrt{A_{11}}\right) \frac{r+\delta}{(h-2 r+\delta)(2 r+\delta)}\right\}
\end{gathered}
$$

$A_{11}, A_{22}, A_{33}$ in Eq. (7) are the Hamaker constants for particle-particle interaction, solventsolvent interaction and polymer-polymer interaction, respectively. The Hamaker constants used for the calculation are $A_{11}=0.85 \times 10^{-2033}, A_{22}=3.7 \times 10^{-2043}$, and $A_{33}=8.4 \times 10^{-2044}$. 

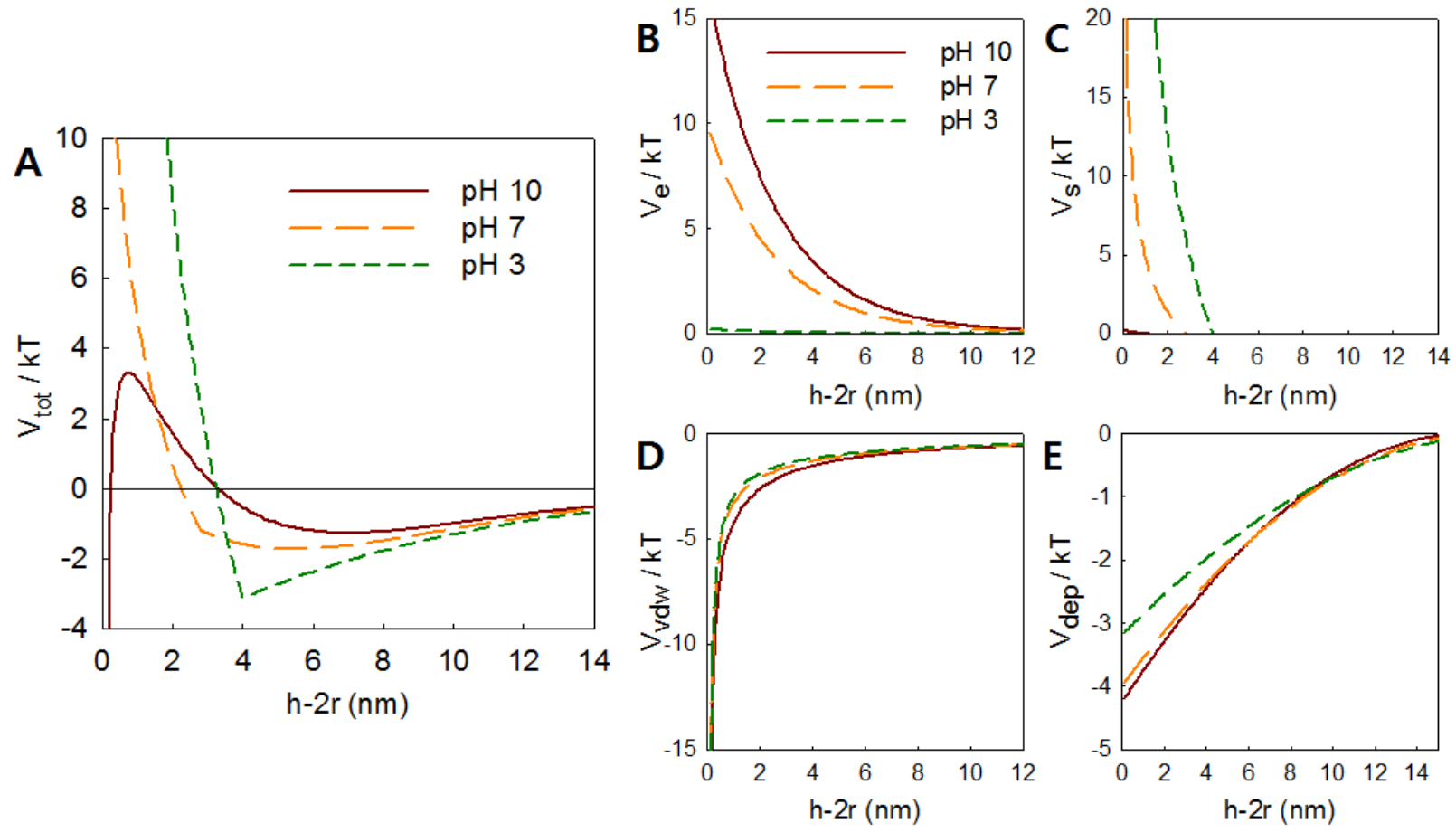

Figure 11. Particle interaction potentials of the silica/PVA suspensions at different $\mathrm{pH}$ values for 0 vol \% solvent evaporation (so at the onset of drying). (A) total potential energy $V_{\text {tot. }}$ (B) electrostatic repulsion $V_{\mathrm{e}}$. (C) steric repulsion $V_{\mathrm{s}}$. (D) van der Waals attraction $\mathrm{V}_{\mathrm{vdw}}$. (E) depletion interaction $\mathrm{V}_{\text {dep }}$.

Fig. 11A shows the total potential energy $\mathrm{V}_{\text {tot }}$ of the silica/PVA mixtures, obtained as the sum of the single potential energies in Figs. 11B to $11 \mathrm{E}$ (i.e. $\mathrm{V}_{\text {tot }}=\mathrm{V}_{\mathrm{dep}}+\mathrm{V}_{\mathrm{s}}+\mathrm{V}_{\mathrm{e}}+\mathrm{V}_{\mathrm{vdw}}$ ), calculated at the onset of drying ( 0 vol \% solvent evaporation). From this it becomes obvious that for $\mathrm{pH} 10$ an energy barrier of $\sim 3 k T$ observed in $\mathrm{V}_{\text {tot }}$ originates from $\mathrm{V}_{\mathrm{e}}$ (Fig. 11B), implying particles are charge-stabilized in the initial liquid state. $\mathrm{V}_{\text {tot }}$ at $\mathrm{pH} 7$ shows a positive wall as $h \rightarrow 2 r$, arising from the combined potentials of $\mathrm{V}_{\mathrm{e}}$ (Fig. 11B) and $\mathrm{V}_{\mathrm{s}}$ (Fig. 11C). A minimum is observed at larger distance $h$, but the range is broad and the depth is below $\mid 2$ $k T$, so that it will not play a role as an attractive minimum. Therefore, particles at $\mathrm{pH} 7$ are expected to be electrostatically and sterically stabilized in the initial liquid state. The 
suspension at $\mathrm{pH} 3$ shows a potential minimum of $\sim|3 k T|$ around $h-2 r=4 \mathrm{~nm}$, followed by a potential wall as $h \rightarrow 2$ r, suggesting that particles can be trapped at this distance of $4 \mathrm{~nm}$, but are strongly sterically stabilized at shorter $h$. The potential minimum outside the steric wall is a result of $V_{v d w}$ and $V_{\text {dep }}$, whereas $V_{e}$ can be ignored. The presence of both an attractive minimum and a positive wall for $h \rightarrow 2 r$ shows a good consistency with the analysis of the liquid structure at pH 3 in Fig. 2, indicating that indeed particles are sterically stabilized at short-range, but depletion flocculated at longer ranges.
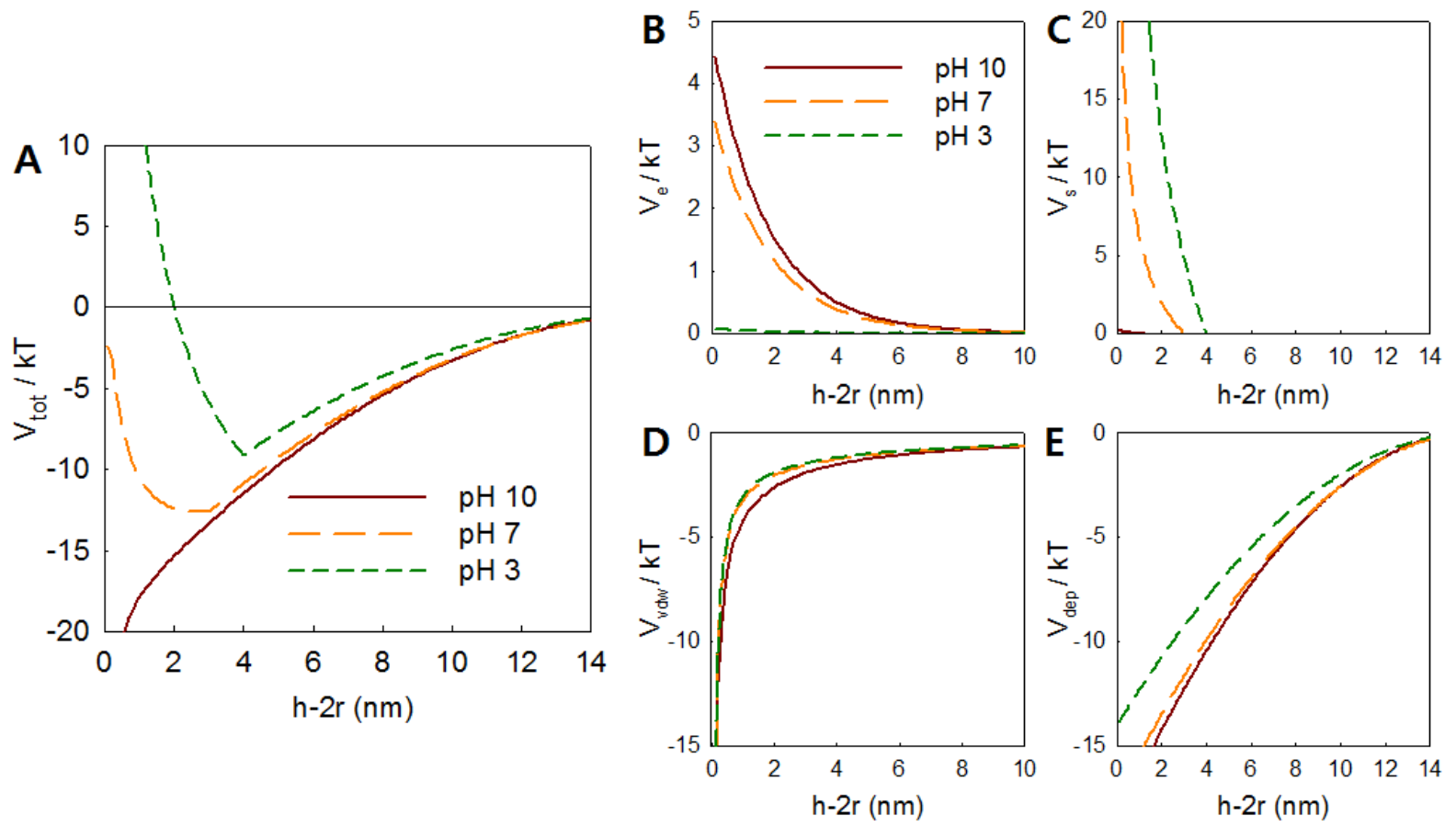

Figure 12. Particle interactions of the silica/PVA suspensions at varying $\mathrm{pH}$ when $50 \mathrm{vol} \%$ solvent evaporates. (A) total potential energy $V_{\text {tot. }}$ (B) electrostatic repulsion $V_{e}$. (C) steric repulsion $\mathrm{V}_{\mathrm{s}}$. (D) van der Waals attraction $\mathrm{V}_{\mathrm{vdw}}$. $(\mathrm{E})$ depletion interaction $\mathrm{V}_{\mathrm{dep}}$.

Fig. 12 shows the potential energy after 50 vol \% of the solvent has evaporated. Solvent evaporation increases both the salt and the polymer concentration in the medium, which 
results in a weakening of $\mathrm{V}_{\mathrm{e}}$ (Fig. 12B) and strengthening of $\mathrm{V}_{\text {dep }}$ (Fig. 12E) compared to the initial state (Figs. $11 \mathrm{~B}$ and $11 \mathrm{E}$, respectively). As a result, $\mathrm{V}_{\text {tot }}$ at $\mathrm{pH} 10$ shows a disappearance of the energy barrier, causing the particles at $\mathrm{pH} 10$ to fall into a primary minimum during drying, resulting in a permanent aggregation. In contrast to this, $\mathrm{V}_{\text {tot }}$ at $\mathrm{pH} 7$ and $\mathrm{pH} 3$ is still exhibiting an upturn as $h \rightarrow 2 r$ that originates from $\mathrm{V}_{\mathrm{s}}$ (Fig. 12C). At larger distances both $\mathrm{pH} 7$ and 3 are dominated by the depletion potential and, as a result, both $\mathrm{pH}$ exhibit a potential minimum in $\mathrm{V}_{\text {tot }}$ at $h-2 r=3-4 \mathrm{~nm}$. The minimum is deep enough $(>/ 7$ $k T \mid$ ) to cause the particles to aggregate, however, the potential wall at smaller distances for both $\mathrm{pH}$ prevents particle from further aggregation so that they can still locally rearrange. The presence of the potential wall even at later stages of drying represents a marked difference between the suspensions at lower $\mathrm{pH}$ and at $\mathrm{pH} 10$

Calculations of the total potential energies from Eqs. (1), (2), (6) and (7) after 70 vol\% solvent evaporation (See Supporting Information S3) are similar to 50\% evaporation, however, with the difference that, for $\mathrm{pH} 7$, the potential wall for $h \rightarrow 2 r$ has disappeared. The new primary for $\mathrm{pH} 7$ is, however, still much more shallow than for $\mathrm{pH} 10$. This indicates that the particles at $\mathrm{pH} 7$ will also eventually experience a permanent aggregation, but only at a higher particle concentration, so that the resulting clusters are much smaller and the structure is more homogeneous than for $\mathrm{pH} 10$. This explains why the dry film at $\mathrm{pH} 7$ is not totally clear, but exhibits a slight turbidity. For $\mathrm{pH} 3$, the potential minimum slightly shifts to smaller $\mathrm{h}$, however, positive values from steric repulsion prevent permanent aggregation.

The evaluation of potential energies with increasing evaporation shows that the $\mathrm{pH}$ dependent amount of adsorbed polymers plays a key role in the evolution of the interaction potential of silica during drying, in particular the possibility of a persistent steric stabilization even at later drying stages when the particles come into close proximity. As an extreme 
example, $\mathrm{V}_{\text {tot }}$ at $\mathrm{pH} 3$ shows an unchanged location of the potential minimum over the whole drying process ( $h-2 r=4 \mathrm{~nm}$ at Figs. 11A and 12A), indicating that the inter-particle distance between neighboring particles does not change during drying. This again highlights the role of adsorbed polymer which stabilized the particles during drying at the short-range.

\section{Conclusion}

In the present study, we explore the development of NP dispersions during drying in $\mathrm{NP}$ /polymer solution films, with a focus on the interaction between particle and hosting polymer. We use an aqueous dispersion of colloidal silica and poly(vinyl alcohol), altering the affinity between particle and polymer via the $\mathrm{pH}$. We observed the structural evolution of the NP dispersion state during drying in-situ by means of vertical x-ray scattering. For a poor

affinity between NPs and hosting polymer, the suspension exhibits a phase separation during drying caused by an early domination of depletion attraction, resulting in dense and larger aggregates of bare particles. On the other hand, for a high affinity between NP and hosting polymer the suspension exhibits a gradual and homogeneous densification. This is caused by a sufficient steric stabilization that balances the depletion attraction sufficiently long during drying to maintain a constant minimum inter-particles distance. This retains the local mobility of the particle during drying and allows for particle rearrangement into a dense, homogenous and thus transparent dry film.

This possibility to tune the particle interaction and local mobility via the addition of polymer at different $\mathrm{pH}$, and thus the structural evolution during drying into the solid state, has been demonstrated in this paper with apparently peculiar evolutions of the transparency of drying silica/PVA films:

- NPs at pH 10 are charge-stabilized in the suspension, but become destabilized during 
drying by polymer induced depletion destabilization in the up-concentrating suspension, which leads finally to a flocculated during drying, thus a transparent liquid dried into a finally turbid solid state.

- On the other hand, NPs at pH 3 were initially flocculated in the suspension due to low charge stabilization, however, steric stabilization by the polymer during the whole drying process ensured mobility of the NPs allowing rearrangement into a finally uniform distribution, thus a turbid liquid dried into a transparent solid state.

The observed drying behavior of silica/PVA film seems to contradict the common belief that stabilized NPs dry into uniform distribution, whereas destabilized NPs form non-uniform distribution after drying ${ }^{46.47}$. However, the quantitative analysis in the present study (based on in-situ determination of the structural evolution with vertical X-ray scattering and the calculation of the evolution of the interaction potentials) suggests that interparticle interactions can significantly evolve during drying and therefore alter the liquid structure to the point that initially stable suspensions destabilize into the solid structure in the drying silica/PVA film, and vice versa.

It should be noted that the evolution of interparticle interaction and corresponding NP dispersion at different stages of drying could be further understood by modeling of the experimental structure factors ${ }^{41}$, however, this will be a future study of SAXS experiments on drying suspensions.

Our systematic drying study focusing on the evolution of NP dispersion and its role of polymer on drying behavior will provide fundamental insights that enable novel design routes for a wide range of coating formulations.

\section{Acknowledgements}


We thank DESY, Hamburg, Germany for access to the X-ray beam line BW1. K H Ahn acknowledges NRF of Korea for funding through No. 2013R1A2A2A07067387, and K Hyun through No. 2010-0024466. S Kim and C Clasen acknowledge financial support from the ERC Starting Grant No. 203043-NANOFIB and from the Research Foundation Flanders (FWO, project G077916N). We also thank to J W Yoon and J Y Moon for support of the SAXS measurement, and J Vermant and S Y Kim for the interpretation of SAXS intensity.

Supporting Information Available: Absolute Scattering intensities of drying film at $\mathrm{pH}$ 10, descriptions on the weight loss measurement during drying, and the calculation of potential energies at $70 \mathrm{vol} \%$ solvent evaporation are available free of charge via the Internet at http://pubs.acs.org.

Conflict of Interest: The authors declare no competing financial interest. 


\section{References}

1. Narayanamoorthy, B.; Datta, K. K. R.; Eswaramoorthy, M.; Balaji, S. Improved Oxygen Reduction Reaction Catalyzed by Pt/Clay/Nafion Nanocomposite for PEM Fuel Cells. ACS Appl. Mater. Interfaces 2012, 4, 3620-3626.

2. Richards, J. J.; Whittle, C. L.; Shao, G.; Pozzo, L. D. Correlating Structure and Photocurrent for Composite Semiconducting Nanoparticles with Contrast Variation SmallAngle Neutron Scattering and Photoconductive Atomic Force Microscopy. ACS Nano 2014, 8, 4313-4324.

3. Muriithi, B.; Loy, D. A. Processing, Morphology, and Water Uptake of Nafion/Ex situ Stöber Silica Nanocomposite Membranes As a Function of Particle Size. ACS Appl. Mater. Interfaces 2012, 4, 6766-6773.

4. Manuel Stephan, A.; Nahm, K. S. Review on composite polymer electrolytes for lithium batteries. Polymer 2006, 47, 5952-5964.

5. Kim, P.; Doss, N. M.; Tillotson, J. P.; Hotchkiss, P. J.; Pan, M.-J.; Marder, S. R.; Li, J.; Calame, J. P.; Perry, J. W. High Energy Density Nanocomposites Based on Surface-Modified BaTiO3 and a Ferroelectric Polymer. ACS Nano 2009, 3, 2581-2592.

6. Song, Q.; Nataraj, S. K.; Roussenova, M. V.; Tan, J. C.; Hughes, D. J.; Li, W.; Bourgoin, P.; Alam, M. A.; Cheetham, A. K.; Al-Muhtaseb, S. A.; Sivaniah, E. Zeolitic imidazolate framework (ZIF-8) based polymer nanocomposite membranes for gas separation. Energy Environ. Sci. 2012, 5, 8359-8369.

7. $\mathrm{Xu}, \mathrm{L} . ; \mathrm{Gao}, \mathrm{L} . ; \mathrm{He}, \mathrm{J}$. Fabrication of visible/near-IR antireflective and superhydrophobic coatings from hydrophobically modified hollow silica nanoparticles and poly(methyl methacrylate). $R S C A d v . \mathbf{2 0 1 2}, 2,12764-12769$.

8. Janes, D. W.; Moll, J. F.; Harton, S. E.; Durning, C. J. Dispersion Morphology of Poly(methyl acrylate)/Silica Nanocomposites. Macromolecules 2011, 44, 4920-4927. 
9. Jouault, N.; Vallat, P.; Dalmas, F.; Said, S. r.; Jestin, J.; Boué, F. o. Well-Dispersed Fractal Aggregates as Filler in Polymer-Silica Nanocomposites: Long-Range Effects in Rheology. Macromolecules 2009, 42, 2031-2040.

10. Kumar, S. K.; Jouault, N.; Benicewicz, B.; Neely, T. Nanocomposites with Polymer Grafted Nanoparticles. Macromolecules 2013, 46, 3199-3214.

11. Kwon, N. K.; Park, C. S.; Lee, C. H.; Kim, Y. S.; Zukoski, C. F.; Kim, S. Y. Tunable Nanoparticle Stability in Concentrated Polymer Solutions On the Basis of the Temperature Dependent Solvent Quality. Macromolecules 2016, 49, 2307-2317.

12. Jouault, N.; Zhao, D.; Kumar, S. K. Role of Casting Solvent on Nanoparticle Dispersion in Polymer Nanocomposites. Macromolecules 2014, 47, 5246-5255.

13. Zhao, D.; Schneider, D.; Fytas, G.; Kumar, S. K. Controlling the Thermomechanical Behavior of Nanoparticle/Polymer Films. ACS Nano 2014, 8, 8163-8173.

14. Kim, S.; Sung, J. H.; Ahn, K. H.; Lee, S. J. Drying of the Silica/PVA Suspension: Effect of Suspension Microstructure. Langmuir 2009, 25, 6155-6161.

15. Meth, J. S.; Zane, S. G.; Chi, C.; Londono, J. D.; Wood, B. A.; Cotts, P.; Keating, M.; Guise, W.; Weigand, S. Development of Filler Structure in Colloidal Silica-Polymer Nanocomposites. Macromolecules 2011, 44, 8301-8313.

16. Kim, S.; Sung, J. H.; Hur, K.; Ahn, K. H.; Lee, S. J. The effect of adsorption kinetics on film formation of silica/PVA suspension. J. Colloid Interface Sci. 2010, 344, 308-314.

17. Zhao, D.; Ge, S.; Senses, E.; Akcora, P.; Jestin, J.; Kumar, S. K. Role of Filler Shape and Connectivity on the Viscoelastic Behavior in Polymer Nanocomposites. Macromolecules 2015.

18. Sen, D.; Spalla, O.; Taché, O.; Haltebourg, P.; Thill, A. Slow Drying of a Spray of Nanoparticles Dispersion. In Situ SAXS Investigation. Langmuir 2007, 23, 4296-4302.

19. Hu, S.; Rieger, J.; Lai, Y.; Roth, S. V.; Gehrke, R.; Men, Y. In-Situ Observation of 
Drying Process of a Latex Droplet by Synchrotron Small-Angle X-ray Scattering. Macromolecules 2008, 41, 5073-5076.

20. Li, J.; Cabane, B.; Sztucki, M.; Gummel, J.; Goehring, L. Drying Dip-Coated Colloidal Films. Langmuir 2012, 28, 200-208.

21. Sanyal, M.; Schmidt-Hansberg, B.; Klein, M. F. G.; Munuera, C.; Vorobiev, A.; Colsmann, A.; Scharfer, P.; Lemmer, U.; Schabel, W.; Dosch, H.; Barrena, E. Effect of Photovoltaic Polymer/Fullerene Blend Composition Ratio on Microstructure Evolution during Film Solidification Investigated in Real Time by X-ray Diffraction. Macromolecules 2011, 44, 3795-3800.

22. Herzog, G.; Benecke, G.; Buffet, A.; Heidmann, B.; Perlich, J.; Risch, J. F. H.; Santoro, G.; Schwartzkopf, M.; Yu, S.; Wurth, W.; Roth, S. V. In Situ Grazing Incidence Small-Angle X-ray Scattering Investigation of Polystyrene Nanoparticle Spray Deposition onto Silicon. Langmuir 2014, 29, 11260-11266.

23. Narayanan, S.; Wang, J.; Lin, X.-M. Dynamical Self-Assembly of Nanocrystal Superlattices during Colloidal Droplet Evaporation by in situ Small Angle X-Ray Scattering. Phys. Rev. Let. 2004, 93, 135503.

24. Roth, S. V.; Herzog, G.; Körstgens, V.; Buffet, A.; Schwartzkopf, M.; Perlich, J.; Kashem, M. M. A.; Döhrmann, R.; Gehrke, R.; Rothkirch, A.; Stassig, K.; Wurth, W.; Benecke, G.; Li, C.; Fratzl, P.; Rawolle, M.; Müller-Buschbaum, P. In situ observation of cluster formation during nanoparticle solution casting on a colloidal film. J. Phys. Condensed Matter 2011, 23, 254208.

25. Sen, D.; Bahadur, J.; Mazumder, S.; Santoro, G.; Yu, S.; Roth, S. V. Probing evaporation induced assembly across a drying colloidal droplet using in situ small-angle Xray scattering at the synchrotron source. Soft Matter 2014, 10, 1621-1627.

26. Jouault, N.; Dalmas, F.; Boué, F.; Jestin, J. Multiscale characterization of filler 
dispersion and origins of mechanical reinforcement in model nanocomposites. Polymer 2012, $53,761-775$.

27. Kim, S.; Hyun, K.; Kim, Y. S.; Struth, B.; Clasen, C.; Ahn, K. H. Drying of a ChargeStabilized Colloidal Suspension in Situ Monitored by Vertical Small-Angle X-ray Scattering. Langmuir 2013, 29, 10059-10065.

28. Nagy, D. J., Size Exclusion Chromatography of Polyvinyl Alcohol and Polyvinyl Acetate. In Handbook of Size Exclusion Chromatography and Related Techniques, Wu, C., Ed. Marcel Dekker, Inc.: New York, 2004.

29. Tadros, T. F. Adsorption of polyvinyl alcohol on silica at various ph values and its effect on the flocculation of the dispersion. J. Colloid Interface Sci. 1978, 64, 36-47.

30. Kim, S.; Hyun, K.; Moon, J. Y.; Clasen, C.; Ahn, K. H. Depletion Stabilization in Nanoparticle-polymer Suspensions: Multi-Length-Scale Analysis of Microstructure. Langmuir 2015, 31, 1892-1900.

31. Dufresne, E. R.; Corwin, E. I.; Greenblatt, N. A.; Ashmore, J.; Wang, D. Y.; Dinsmore, A. D.; Cheng, J. X.; Xie, X. S.; Hutchinson, J. W.; Weitz, D. A. Flow and Fracture in Drying Nanoparticle Suspensions. Phys. Rev. Let. 2003, 91, 224501.

32. Tirumkudulu, M. S.; Russel, W. B. Cracking in Drying Latex Films. Langmuir 2005, $21,4938-4948$.

33. Russel, W. B.; Saville, D. A.; Schowalter, W. R., Colloidal Dispersions. Cambridge University Press: 1989.

34. Milling, A.; Vincent, B.; Emmett, S.; Jones, A. Depletion flocculation in dispersions of sterically-stabilised silica particles 3 . The effect of grafted chain coverage. Colloids Surf. 1991, 57, 185-195.

35. Vincent, B.; Edwards, J.; Emmett, S.; Jones, A. Depletion flocculation in dispersions of sterically-stabilised particles ("soft spheres"). Colloids Surf. 1986, 18, 261-281. 
36. Vincent, B.; Luckham, P. F.; Waite, F. A. The effect of free polymer on the stability of sterically stabilized dispersions. J. Colloid Interface Sci. 1980, 73, 508-521.

37. Klein, J.; Rossi, G. Analysis of the Experimental Implications of the Scaling Theory of Polymer Adsorption. Macromolecules 1998, 31, 1979-1988.

38. Runkana, V.; Somasundaran, P.; Kapur, P. C. A population balance model for flocculation of colloidal suspensions by polymer bridging. Chem. Eng. Sci. 2006, 61, 182-191. 39. Jones, A.; Vincent, B. Depletion flocculation in dispersions of sterically-stabilised particles 2. Modifications to theory and further studies. Colloids Surf. 1989, 42, 113-138.

40. Asakura, S.; Oosawa, F. On Interaction between Two Bodies Immersed in a Solution of Macromolecules. J. Chem. Phys. 1954, 22, 1255-1256.

41. Hayter, J. B.; Penfold, J. An analytic structure factor for macroion solutions. Mol. Phys 1981, 42, 109 - 118.

42. Otsubo, Y. Effect of polymer adsorption on the rheological behavior of silica suspensions. J. Colloid Interface Sci. 1986, 112, 380-386.

43. Vincent, B. The van der Waals attraction between colloid particles having adsorbed layers. II. Calculation of interaction curves. J. Colloid Interface Sci.1973, 42, 270-285.

44. Lyklema, J.; van Vliet, T. Polymer-stabilized free liquid films. Faraday Discuss. Chem. Soc 1978, 65, 25 - 32.

45. Koopal, L. K. The unperturbed conformation and interaction parameters of polyvinylalcohol in aqueous solutions. Polydispersity effects. Colloid Polym. Sci. 1981, 259, $490-498$.

46. Guo, J. J.; Lewis, J. A. Aggregation Effects on the Compressive Flow Properties and Drying Behavior of Colloidal Silica Suspensions. Journal of the American Ceramic Society 1999, 82, 2345-2358. 
47. Singh, K. B.; Bhosale, L. R.; Tirumkudulu, M. S. Cracking in Drying Colloidal Films of Flocculated Dispersions. Langmuir 2009, 25, 4284-4287. 


\section{Graphical table of contents}

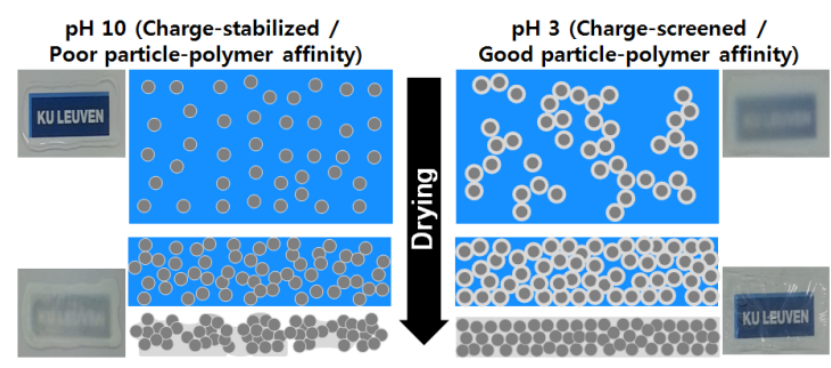

